\title{
Diffusive Stability of Spatial Periodic Solutions of the Swift-Hohenberg Equation
}

\author{
Guido Schneider \\ IfAM, Universität Hannover, Welfengarten 1, D-30167 Hannover, Germany
}

Received: 1 September 1995/Accepted: 6 October 1995

\begin{abstract}
We are interested in the nonlinear stability of the Eckhaus-stable equilibria of the Swift-Hohenberg equation on the infinite line with respect to small integrable perturbations. The difficulty in showing stability for these stationary solutions comes from the fact that their linearizations possess continuous spectrum up to zero. The nonlinear stability problem is treated with renormalization theory which allows us to show that the nonlinear terms are irrelevant, i.e. that the fully nonlinear problem behaves asymptotically as the linearized one which is under a diffusive regime.
\end{abstract}

\section{Introduction}

We consider evolutionary problems on the infinite line. Here we are interested in the nonlinear stability of the bifurcating, spatial periodic, stationary solutions $u_{0}$ of the Swift-Hohenberg equation with respect to small integrable, non-periodic perturbations. Under stability we understand the following generalization of the usual stability definition $\left(\mathscr{B}_{1}=\mathscr{B}_{2}\right)$.

Definition 1. Let $\mathscr{B}_{1}, \mathscr{B}_{2}$ be Banach spaces and let $S_{t}$ be an evolution operator. $A$ fixed point $u_{0}=S_{t} u_{0}$ is called $\left(\mathscr{B}_{1}, \mathscr{B}_{2}\right)$-stable under $S_{t}$ if the following holds: For all $\mu>0$ there exists a $\delta>0$ such that from $\left\|v-u_{0}\right\|_{\mathscr{B}_{1}}<\delta$ follows that $\left\|S_{t} v-u_{0}\right\|_{\mathscr{B}_{2}}<\mu$ for all $t \geqq 0$. The point $u_{0}$ is called asymptotically $\left(\mathscr{B}_{1}, \mathscr{B}_{2}\right)$ stable if additionally $\lim _{t \rightarrow \infty} S_{t} v=u_{0}$ in $\mathscr{B}_{2}$ holds.

The first step in stability proofs is the examination of the spectrum of the linearization around $u_{0}$. As always for translationally invariant problems the linearization around the nontrivial ground state $u_{0}$ possesses $\partial_{x} u_{0}$ as eigenvector to the eigenvalue zero. Since zero is the maximal eigenvalue and no Jordan-block appears the stability question has to be answered through the nonlinear terms. In cases of an appearing spectral gap between zero and the negative eigenvalues center manifold theory applies to prove nonlinear stability. The center manifold is usually equal to the family of stationary solutions $\left\{u_{0}(s+\cdot) \mid s \in \mathbb{R}\right\}$. Thus, asymptotic phase theory $[\mathrm{He} 81]$ predicts that every solution nearby the center manifold converges to 
exactly one equilibrium. This theory applies for some problems on the infinite line ([He81, PW94, Ha94]) or if we assume in our problem spatial periodicity.

Under small non-periodic, integrable, perturbations the linearization of our problem around the ground-state $u_{0}$ possesses continuous spectrum up to zero without any spectral gap. Therefore center manifold theory is not applicable. The most powerful existing tool to handle such problems is the method of diffusive stability. The simplest example, where this method applies, is the nonlinear stability of $u \equiv 0$ in $\partial_{t} u=u_{x x}+u^{p}$ with $x \in \mathbb{R}$ and $p>3$. Solutions to initial conditions in $L^{\infty}$ can explode in finite time, but solutions to initial conditions $\left.u\right|_{t=0}$ with $\left\|\left.u\right|_{t=0}\right\|_{L^{1} \cap L^{\infty}}<\delta$ stay small in $L^{1} \cap L^{\infty}$ for all times, if $\delta>0$ is sufficiently small and $p>3$ (cf. [CEE92]). The solutions tend to zero as $t^{-1 / 2}$ in $L^{\infty}$ and stay bounded in $L^{1}$ for $t \rightarrow \infty$. Since this is the same behavior as for the linearized equation, one says that the nonlinearity is asymptotically irrelevant for this problem. This method was used in [CEE92, CE92, Ka94] to show for instance the diffusive stability of the Eckhausstable spatial periodic solutions in the real (i.e. real coefficients) Ginzburg-Landau equation. The same idea of irrelevant nonlinearities is also used in [GJK93] for the stability proof of viscous shock profiles for a different linearized problem.

Let us introduce the spaces $H^{m}(n)$. A function is in $H^{m}(n)$ if $x \mapsto(1+$ $\left.x^{2}\right)^{n / 2} u(x)$ is in $H^{m}$. Perturbations $u(\cdot, 0) \in H^{2}(2)$ for linear one-dimensional diffusion problems $\partial_{t} u=u_{x x}$ behave asymptotically as

$$
\sup _{x}\left|u(x, t)-\frac{1}{2 \sqrt{\pi t}} U e^{-x^{2} /(4 t)}\right| \leqq \mathcal{O}(1 / t), \quad \text { for } t \rightarrow \infty
$$

with $U=\left.\int u\right|_{t=0} d x$. From this follows the $\left(H^{2}(2), L^{\infty}\right)$-stability of $u \equiv 0$. So-called renormalization theory allows us to show this behavior also for the solutions of the nonlinear problem $\partial_{t} u=u_{x x}+u^{p}$ under the above restrictions, where now $U=$ $U\left(\left.u\right|_{t=0}\right)$. Simply said the renormalized solutions $(2 \sqrt{\pi t}) u(x \sqrt{t}, t)$ converge towards $U e^{-x^{2} / 4}$. In [BK92] this method is used to show the asymptotic behavior for the perturbations of the stationary solutions and in [BK94, Ga94] for the marginal stable front solutions of the Ginzburg-Landau equation.

Different approaches for the stability proof of the front solutions are used in [Ki92, EW94]. In [Wa94] the invariant manifold approach is made applicable for diffusive stability problems by rescaling the solutions.

The Ginzburg-Landau equation appears as a so-called modulation equation in the description of more complicated systems, for instance for the description of pattern formation in Bénard's problem [NW69] or in the Taylor-Couette problem. It can be derived for weakly unstable systems for which the linear stability analysis predicts a pattern of almost spatial periodic nature. In the last years approximation properties ([CE90b, vH91, Sch94b]), attractivity of the so described set of solutions ([Eck93, Sch95]), and uppersemicontinuity of the original system attractor towards the Ginzburg-Landau attractor ([MS95]) have been shown. So it is natural to ask if the above stability results which have been obtained for the modulation equation also hold for the associated original systems.

Recent papers have shown that good model problems for the mathematical examination of the Ginzburg-Landau formalism are the Swift-Hohenberg and the Kuramoto-Shivashinsky equation. Thus, we consider here the nonlinear diffusive stability of the spatial-periodic stationary solutions of the Swift-Hohenberg equation. Moreover, we are interested in the asymptotic behavior of their perturbations. 
For this problem the same main difficulties appear as for the hydrodynamical problems, but with less technical details.

The Swift-Hohenberg equation

$$
\partial_{t} u=-\left(1+\partial_{x}^{2}\right)^{2} u+\varepsilon^{2} u-u^{3}, \quad(t \geqq 0, x \in \mathbb{R})
$$

possesses for small $\varepsilon^{2}>0$ a three-parametric family of stationary solutions

$$
u_{0}(\omega, \varphi, \varepsilon)[x]=\varepsilon\left(\sqrt{1-4 \omega^{2}} / \sqrt{3}\right) e^{i(1+\varepsilon \omega) x} e^{i \varphi}+c . c+\mathcal{O}\left(\varepsilon^{2}\right)=\varepsilon \tilde{u}_{0}
$$

with $u_{0}(\omega, \varphi, \varepsilon)[x]=u_{0}(\omega, \varphi, \varepsilon)[x+2 \pi /(1+\varepsilon \omega)]$ which bifurcate for $\varepsilon=0$ from $u \equiv 0$. Since [Eck65] it is known that the solutions $u_{0}(\omega, \varphi, \varepsilon)$ with $\left|4 \omega^{2}\right|<1 / 3+$ $\mathcal{O}(\varepsilon)$ are marginally spectral-stable, i.e. that the linearization around these solutions possesses the spectrum $(-\infty, 0]$. Therefore, these solutions are called Eckhausstable. The spectrally unstable solutions are called Eckhaus- or sideband-unstable.

For three decades it was an open question if these solutions are really nonlinear stable on the infinite line under small non-periodic, integrable, perturbations. Our main result is as follows.

Theorem 2. There exists a $\varepsilon_{0}>0$ such that the following holds. Fix $\omega, \varphi$, and $\varepsilon \in\left(0, \varepsilon_{0}\right)$ such that $u_{0}=u_{0}(\omega, \varphi, \varepsilon)$ is an Eckhaus-stable stationary solution of (2). Then there exists a strictly positive constant $C_{1}(\omega, \varepsilon)$ such that the following holds. Let $u_{0}+\left.v\right|_{t=0}$ be an initial condition for (2), where $\left.v\right|_{t=0}$ is sufficiently small in a later on described space $\mathscr{B}$ of initial conditions. Then the perturbation $v$ behaves asymptotically as

$$
v(x, t)=\frac{1}{\sqrt{t}} \tilde{A} e^{-x^{2} /\left(4 C_{1}(\omega, \varepsilon) t\right)} \partial_{x} \tilde{u}_{0}(\omega, \varphi, \varepsilon)[x]+\mathcal{O}\left(t^{p-1}\right) \quad \text { for } t \rightarrow \infty
$$

and each $p \in(0,1 / 2)$ in $L^{\infty}$. Here $\tilde{A} \in \mathbb{R}$ is a constant which only depends on the initial conditions. From this follows the $\left(\mathscr{B}, L^{\infty}\right)$-stability of $u_{0}$. We have the asymptotics $C_{1}(\omega, \varepsilon)=\mathcal{O}(1)$ and $\tilde{A}=\mathcal{O}(1)$ for $\varepsilon \rightarrow 0$.

The exact result is formulated in Theorem 15 and in the following corollaries. This asymptotic behavior is exactly what is expected after combining the approximation result (cf. [vH91]) for the Ginzburg-Landau approximation with the diffusive stability result ([BK92]) for the associated solutions of the Ginzburg-Landau equation. See (26). Unfortunately this combination does not allow us to prove Theorem 2. Our proof is essentially based on an explicit representation of the linearisation around $u_{0}$. The functional analytic set-up is similar to the one for the linear Schrödinger equation with spatial periodic potential. Therefore our method does not allow us at the moment to approach the nonlinear stability of the marginal stable front solutions of the Swift-Hohenberg equation or to transfer further results of [BK92] to the Swift-Hohenberg problem. The first problem would transfer the result of [BK94,Ga94] to an original system, but it is expected to be much more complicated. This will be the subject of further research. For the linear stability analysis of this problem see [CE87]. Generalizations of the results stated here to real applications as there are Bénard's problem or the Taylor-Couette problem are planned in future work. Since the mathematical theory of the Ginzburg-Landau formalism for these problems is already developed in [Sch94b] we do not expect conceptual difficulties in transferring the above result from the Swift-Hohenberg 
equation to the hydrodynamical applications, although the functional analytic set-up for real applications becomes more complicated.

The plan of the paper is as follows. In the next section we compute the spectrum. Then we introduce the Ginzburg-Landau formalism and renormalization theory. In the fourth section we prove the nonlinear stability result for the Eckhaus-stable stationary solutions of the Swift-Hohenberg equation.

\section{Spectral Properties}

The first step in stability proofs is always the examination of the linearized problem. This problem is already treated in several papers (cf. [Eck65, CE87, CE90a]). Since the linear stability analysis shows us how to handle the nonlinear problem we recall it. Additional to the spectral properties we gain a useful representation of the linear semigroup which allows us to analyse the asymptotic behavior of the perturbations. Our presentation of this subject is based on [Mi94]. In favor of clarity we consider first the spectral stability of $u_{0}(0,0, \varepsilon)$ which is simply denoted by $u_{0}$. We take the solution $u_{0}$ as the new origin of the Swift-Hohenberg equation and obtain

$$
\partial_{t} v=\mathscr{M} v+N_{1}(v)
$$

with

$$
\mathscr{M} v=-\left(1+\partial_{x}^{2}\right)^{2} v+\varepsilon^{2} v-3 u_{0}^{2} v \quad \text { and } \quad N_{1}(v)=-3 u_{0} v^{2}-v^{3} .
$$

Linearizing (3) around $v \equiv 0$ gives the eigenvalue problem

$$
\mathscr{M} v=\mu v \text {. }
$$

We are looking for $\mu \in \mathbb{C}$ such that (4) possesses non-trivial uniformly bounded solutions $v$. The first observation is made by considering (4) in Fourier space. The space of functions with wave-number support $Z_{l}=\{k=m+l \mid m \in Z\}$ is invariant under the action of $\mathscr{M}$, where $l \in \mathbb{R}$ is a fixed real number. Since $Z_{l}$ and $Z_{l+1}$ coincide it suffices to restrict to $l \in[-1 / 2,1 / 2)$. So it is natural to search for eigenfunctions in these invariant subspaces. Since every wave-number is contained in exactly one $Z_{l}$ we can expect that the constructed eigenfunctions span the whole space.

In physical space the invariance of $Z_{l}$-supported functions implies that $\mathscr{M}$ leaves the space of functions of the form $w_{l}(x) e^{l l x}$ with $w_{l}(x)=w_{l}(x+2 \pi)$ invariant. Inserting this into (4) gives

$$
\mathscr{M}_{l} w_{l}:=-\left(1+\left(i l+\partial_{x}\right)^{2}\right)^{2} w_{l}+\varepsilon^{2} w_{l}-3 u_{0}^{2} w_{l}=\mu w_{l} .
$$

For fixed $l$ this problem is self-adjoint for $w_{l} \in L_{\text {per }}^{2}(0,2 \pi)$. Therefore, all eigenvalues are real. The operator $\mathscr{M}_{l}: H_{\text {per }}^{4} \rightarrow L_{\text {per }}^{2}$ has a compact resolvent $\left(\mathscr{M}_{l}-\mu I d .\right)^{-1}$ for some $\mu \in \mathbb{C}$. From this follows the existence of a discrete set of eigenvalues

$$
\left\{\mu_{j}\left(\varepsilon^{2}, l\right) \in \mathbb{C} \mid j \in \mathbb{N}, \mu_{j} \geqq \mu_{j+1} \rightarrow-\infty \text { for } j \rightarrow \infty\right\}
$$

and a corresponding set of eigenfunctions $\left\{f_{j}\left(\varepsilon^{2}, l\right) \mid j \in \mathbb{N}\right\}$ for fixed $l$. We normalize $f_{j}$ such that $\left\|f_{j}\left(\varepsilon^{2}, l, \cdot\right)\right\|_{L^{2}(0,2 \pi)}=1$. 
It remains to compute these eigenvalues. It is easy to see that for fixed $l_{1}>0$, there exist $\varepsilon_{0}, \sigma_{0}\left(l_{1}\right)>0$ such that for all $\varepsilon \in\left(0, \varepsilon_{0}\right)$ and all $|l|>l_{1}$ all eigenvalues fulfill $\mu_{j}(l)<-\sigma_{0}$. For $l=\varepsilon=0$ the eigenvalue problem can be solved explicitly. We obtain a two-dimensional subspace corresponding to the zero eigenvalue. All other eigenvalues fulfill again $\mu_{j}(l)<-\sigma_{0}=\mathcal{O}(1)$. Therefore $\mathscr{G}(l, \varepsilon, \mu) w=\mathscr{M}(l, \varepsilon) w-\mu w=0$ has to be solved with respect to $w(l, \varepsilon)$ for $l, \varepsilon, \mu$ small. In [Mi94] this problem is handled as a bifurcation problem. We always have the trivial solution $w=0$ for all $l, \mu$, and $\varepsilon$. Solutions can bifurcate from this trivial branch if $\left(\partial_{w} \mathscr{G}(l, \varepsilon, 0)\right)^{-1}$ does not exist and so the implicit function theorem cannot be applied to continue the trivial branch. We know already that $\partial_{w} \mathscr{G}(0,0,0)=\mathscr{M}(0,0)$ is not invertible and has a two-dimensional kernel spanned by $U_{1}=\sin x$ and $U_{2}=\cos x$. Thus we apply the method of LyapunovSchmidt to compute the bifurcating solutions. Let $P$ be the orthogonal projection on this kernel and let $w=a U_{1}+b U_{2}+\mathscr{V}$ with $(1-P) \mathscr{V}=\mathscr{V}$. The hyperbolic part $(1-P) \mathscr{G}(l, \varepsilon, \mu, a, b, \mathscr{V})=0$ possesses the resolution $\mathscr{V}=\mathscr{V}(l, \varepsilon, \mu, a, b)$. Inserting this into $P \mathscr{G}(l, \varepsilon, \mu, a, b, \mathscr{V})=0$ gives the bifurcation equation

$$
\begin{aligned}
& {\left[\left(\begin{array}{cc}
\rho-\mu & -i \delta \\
i \delta & \rho+c(\varepsilon)-\mu
\end{array}\right)+\mathcal{O}\left(\varepsilon^{4}\right)\left(\begin{array}{cc}
\mathcal{O}\left(l^{2}+|\mu|\right) & \mathcal{O}(|l|+|\mu|) \\
\mathcal{O}(|l|+|\mu|) & \mathcal{O}\left(l^{2}+|\mu|\right)
\end{array}\right)\right]\left(\begin{array}{l}
a \\
b
\end{array}\right)} \\
& \quad=\mathscr{G}_{1}\left(\begin{array}{l}
a \\
b
\end{array}\right)=0
\end{aligned}
$$

with

$$
\rho=-4 l^{2}-l^{4}, \quad \delta=-4 l^{3}, \quad c(\varepsilon)=-2 \varepsilon^{2}+\mathcal{O}\left(\varepsilon^{4}\right) .
$$

Computing $\mu_{1,2}(l, \varepsilon)$ in such a way that the determinant of $\mathscr{G}_{1}$ vanishes, gives the curve of eigenvalues $\mu_{1}$ and $\mu_{2}$.

Lemma 3. There exist $l_{0}, \varepsilon_{0}>0$ such that for all $|l| \leqq\left|l_{0}\right|$ and $\varepsilon \in\left[0, \varepsilon_{0}\right]$ the eigenvalues $\mu_{1,2}$ have the following expansion:

$$
\mu_{1}\left(\varepsilon^{2}, l\right)=-c_{1}(\varepsilon) l^{2}+\mathcal{O}\left(l^{4}\right), \quad \mu_{2}\left(\varepsilon^{2}, l\right)=-2 \varepsilon^{2}-c_{1}(\varepsilon) l^{2}+\mathcal{O}\left(l^{4}+\varepsilon^{4}\right),
$$

where $c_{1}(\varepsilon)=4+\mathcal{O}\left(\varepsilon^{2}\right)$.

Corresponding to these curves of eigenvalues is a family of eigenfunctions $f_{1,2}\left(\varepsilon^{2}, l\right)$. They have the following useful property.

Lemma 4. Fix $\omega, \varphi$ and $\varepsilon$. Then the eigenfunction $f_{1}$ can be expanded as $f_{1}(l)=$ $g_{0}+i l g_{1}+\mathcal{O}\left(l^{2}\right)$, where $g_{0}, g_{1} \in H^{2}(0,2 \pi)$ are real-valued functions.

Proof. We have $g_{0}=\partial_{x} u_{0} /\left\|\partial_{x} u_{0}\right\|_{L^{2}(0, \pi)}$. The operator $\mathscr{M}_{l}$ is of the form $\mathscr{M}_{l}=$ $A+i l B+\mathcal{O}\left(l^{2}\right)$, where $A$ and $B$ are real operators. This holds also for general $u_{0}(\omega, \varphi, \varepsilon)$. For this see [Mi94]. The eigenvalues $\mu_{1}$ fulfills $\mu_{1}(l)=\mathcal{O}\left(l^{2}\right)$. Let $h_{1} \in$ $L^{2}(0,2 \pi)$ also be real-valued. Inserting the ansatz $f_{1}(l)=g_{0}+i l g_{1}+l h_{1}+\mathcal{O}\left(l^{2}\right)$ into $\mathscr{M}_{l} f_{1}(l)=\mu_{1}(l) f_{1}(l)$ gives for the $l^{1}$-order terms: $i A g_{1}+A h_{1}+i B g_{0}=0$. Thus $h_{1} \in \operatorname{ker} A$, i.e. $h_{1} \| g_{0}$ or $h_{1}=\beta \partial_{x} u_{0}$ for a $\beta \in \mathbb{R}$. Since we have normalized $f_{1}$ we have to choose $\beta=0$.

Note that the normalization plays no role in the proof of (33). Summarizing our result gives the following picture. 


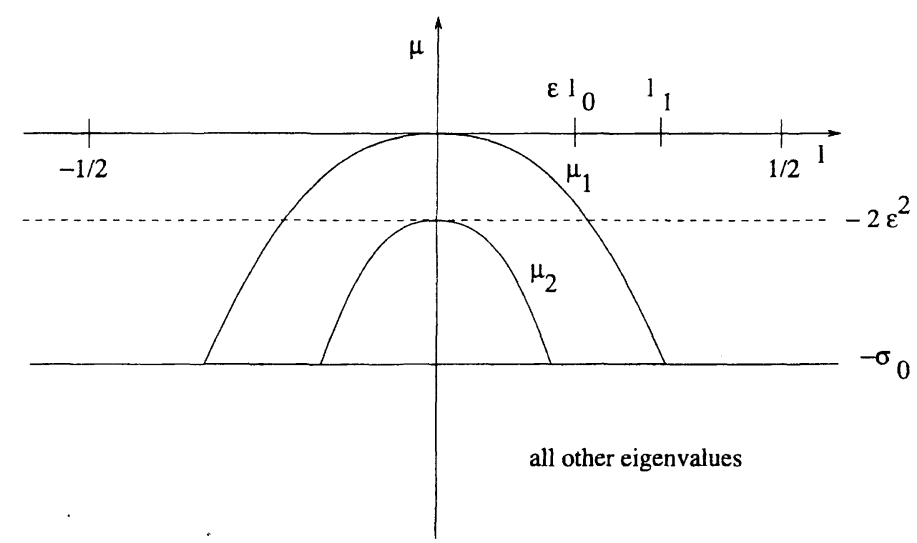

Fig. 1.

Remark. 5. For general $u_{0}(\omega, \varphi, \varepsilon)$ the action of $\mathscr{M}$ leaves the space of functions with Fourier support $Z_{l}^{1+\varepsilon \omega}=\{k=(1+\varepsilon \omega)(m+l) \mid m \in Z\}$ invariant. So the same procedure works. The picture of eigenvalues looks like that above for all Eckhaus-stable solutions $u_{0}(\omega, \varphi, \varepsilon)$ if $4 \omega^{2}<1 / 3+\mathcal{O}(\varepsilon)$. In general the critical eigenvalue looks like $\mu_{1}(l) \sim-C_{1}(\omega, \varepsilon) l^{2}+\ldots$, where $0<C_{1}(\omega, \varepsilon) \rightarrow 0$ for $4 \omega^{2} \rightarrow 1 / 3+\mathcal{O}(\varepsilon)$. See [Mi94].

Until now we have shown spectral stability. Before we establish the nonlinear stability result for the Swift-Hohenberg equation, let us explain the existing result for the Ginzburg-Landau equation. This will make clear what has to be done.

\section{Ginzburg-Landau Formalism and Renormalization Theory}

In this section we give a brief introduction to the so-called Ginzburg-Landau formalism and to renormalization theory. As already said the Ginzburg-Landau equation can be derived as a modulation equation for the Swift-Hohenberg equation. The existing diffusive stability results for the stationary solutions of the GinzburgLandau equation have motivated us to show a similar result for an original system. Therefore it is natural to compare the two stability results. Hence the GinzburgLandau formalism is introduced first. To establish the nonlinear stability result we use renormalization theory. The application of this method is explained for the simplest possible model problem. If you are familiar with the Ginzburg-Landau formalism and renormalization theory you can skip the following section.

3.1. Ginzburg-Landau Formalism. In this paper we start the investigation with a simple model problem in one space dimension. In future work we will treat generalizations to hydrodynamical applications, as there are Bénard's problem in a strip or the Taylor-Couette problem. The simplest example where the GinzburgLandau formalism applies is the Swift-Hohenberg equation (2) we consider. Linearizing (2) around $u \equiv 0$ gives solutions of the form $v(x, t)=e^{\hat{\lambda} t+i k x}$, where $\hat{\lambda}\left(k, \varepsilon^{2}\right)=-\left(1-k^{2}\right)^{2}+\varepsilon^{2}$. One observes that $\hat{\lambda}\left(k, \varepsilon^{2}\right)$ is positive for $k$ close to \pm 1 
with height $\mathcal{O}\left(\varepsilon^{2}\right)$ and width $\mathcal{O}(\varepsilon)$. Therefore the stability curve is given by the following picture:

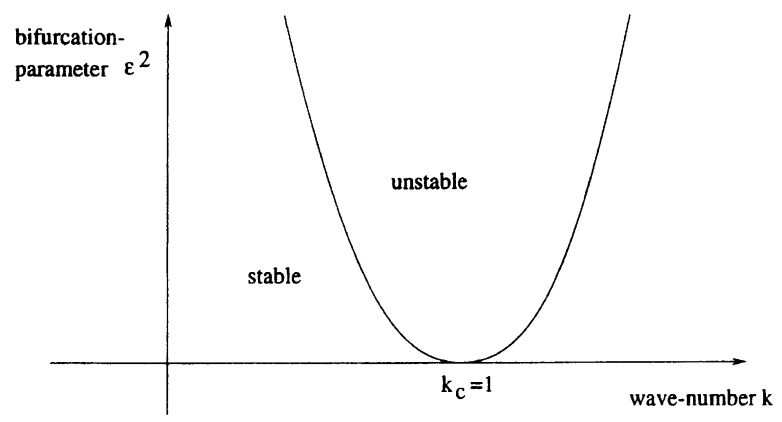

Fig. 2 .

The same stability curve appears for many problems, in particular for the above mentioned hydrodynamical problems. Due to the stability curve, it is natural to expect that for small $\varepsilon^{2}>0$ the long-time behavior of (2) is described by bifurcating solutions $u$, which behave approximately as

$$
u=\widetilde{\psi}_{\varepsilon}(A):=\varepsilon A(X, T) e^{i x}+\varepsilon \bar{A}(X, T) e^{-i x},
$$

with slow time scale $T=\varepsilon^{2} t$ and large spatial scale $X=\varepsilon x$. This ansatz for $u$ is the starting point of the Ginzburg-Landau formalism, and formally equating equal powers in $\varepsilon$ and $e^{i x}$ to zero shows that the complex valued amplitude $A=A(X, T) \in$ $\mathbb{C}$ should satisfy a Ginzburg-Landau equation

$$
\partial_{T} A=4 \partial_{X}^{2} A+A-3|A|^{2} A .
$$

Recent work (see [CE90b, KSM92, vH91, Sch94a, Sch94b]) has demonstrated that the Ginzburg-Landau formalism provides a valid approximation in the sense that $\widetilde{u}(t, x)=\widetilde{\psi}_{\varepsilon}(A)$ approximates a true solution $u$ of (2), whenever $A$ solves (7). Moreover, in [Eck93, Sch94c, Sch95] the attractivity property of the set of solutions in the form (6) was shown. The original system is only reflected in the coefficients of the Ginzburg-Landau equation which can be complex-valued.

3.2. The Diffusive Stability Result for the Ginzburg-Landau Equation. In polar coordinates $A(X, T)=r(X, T) e^{i \phi(X, T)}$ the Ginzburg-Landau equation (7) is given by

$$
\begin{aligned}
& \partial_{T} r=4 r_{X X}-4\left(\partial_{X} \phi\right)^{2} r+r-3 r^{3}, \\
& \partial_{T} \phi=4 \phi_{X X}+8 r_{X} \phi_{X} / r .
\end{aligned}
$$

It is easy to see that $(r, \phi)=\left(\sqrt{1-4 \omega^{2}} / \sqrt{3}, \omega x+\phi_{0}\right)$ defines a two-parametric family of stationary solutions $A_{0}\left(\omega, \phi_{0}\right)=\sqrt{\left(1-4 \omega^{2}\right) / 3} e^{l\left(\omega x+\phi_{0}\right)}$. The linearization around a member of this family $(r, \phi)=\left(\sqrt{1-4 \omega^{2}} / \sqrt{3}+s, \omega x+\phi_{0}+\psi\right)$ possesses eigenfunctions $(s, \psi)=\left(s_{l} e^{i l x}, \psi_{l} e^{i l x}\right)$ and corresponding eigenvalues

$$
\lambda_{1,2}(\omega, l)=-4 l^{2}-\left(1-4 \omega^{2}\right) \pm \sqrt{\left(1-4 \omega^{2}\right)^{2}+64 \omega^{2} l^{2}} .
$$


Since $\lambda_{1}(\omega, 0)=0$ and $\partial_{l} \lambda_{1}(\omega, 0)=0$ (which follows from $\lambda_{1}(\omega, l)=\lambda_{1}(\omega,-l)$ ), it is clear that the sign of $\partial_{l}^{2} \lambda_{1}(\omega, 0)$ answers the question about positive eigenvalues. It is easy to compute that for $4 \omega^{2}>1 / 3$ instability occurs.

Let us consider $\omega=0$ which corresponds to $\omega=0$ in (2). Here we have $\lambda_{1}=-4 l^{2}$ and $\lambda_{2}=-2-4 l^{2}$. These eigenvalues correspond to $\mu_{1}$ and $\mu_{2}$ in (2). Recalling the above Ginzburg-Landau scalings shows again that $\mu_{2}$ in (2) can only be negative of order $\mathcal{O}\left(\varepsilon^{2}\right)$.

Now we are at the same point as we are for the Swift-Hohenberg problem. The linearization possesses continuous spectrum up to zero, so stability is determined by the nonlinear terms. In our PDE-situation it is nearly impossible to conclude stability from the form of the nonlinearity, but see also [EW94]. Therefore we need a method which works without much knowledge of the nonlinear terms.

The coordinates corresponding to $\lambda_{2}$ are exponentially damped. So all difficulties come from the curve $\lambda_{1}=-4 l^{2}$. A similar curve $\tilde{\lambda}_{1}=-k^{2}$ appears after Fourier transforming $u \mapsto \mathscr{F} u=\hat{u}=\frac{1}{2 \pi} \int u(x) e^{-i k x} d x$, the heat equation $\partial_{t} u=u_{x x}$ on the real line. For this equation we have the asymptotic behavior

$$
\sup _{x}\left|\sqrt{t} u(x \sqrt{t}, t)-\sqrt{\pi} \hat{u}(0,0) e^{-x^{2} / 4}\right|=\mathcal{O}(1 / \sqrt{t}),
$$

for $\left.u\right|_{t=0} \in H^{2}(2)$. Since the curves $\lambda_{1}$ and $\tilde{\lambda}_{1}$ are both parabolas, the same behavior can be proved for the perturbations of the linearization around the stationary solutions of the Ginzburg-Landau equation. Looking more closely to the proof of this linear result shows that only the asymptotic parabola shape of $\lambda_{1}$ at the wavenumber $l=0$ plays an important role and not the fact that a diffusion equation is considered. Therefore one can expect that due to the form of $\mu_{1}$ a similar result holds for $\partial_{t} v=\mathscr{M} v$.

For the nonlinear stability let us consider first the nonlinear diffusion equation $\partial_{t} u=u_{x x}+u^{p}$. It is known that $u \equiv 0$ is classical stable in $L^{1} \cap L^{\infty}$, whenever $p>3$. In [CEE92] this idea was transferred to the stability question for the stationary solutions of the Ginzburg-Landau equation. With the renormalization approach ([BK92]) the asymptotic behavior (9) for the perturbations and the $\left(H^{2}(2), L^{\infty}\right)$ stability can be shown for the nonlinear problems, too.

Although center manifold theory does not apply for our stability problem, suppose for the moment that we are able to reduce the whole system to a single nonlinear master diffusion equation. We are done if we show that in the nonlinearity all low order terms are missing. Before we explain how renormalization theory allows us to show (9) for the nonlinear problem, let us discuss the question: why should the low order terms vanish?

At first sight this seems to be a miracle. It becomes clear that it is no miracle if we consider the Swift-Hohenberg equation with periodic boundary conditions $u(x)=u(x+2 \pi)$. We know already that the linearization around $u_{0}=u_{0}(0,0, \varepsilon)$ possesses one zero eigenvalue which corresponds to the translation-invariance of the problem. All other eigenvalues are negative and bounded uniformly away from the imaginary axis. (This is the case $l=0$.) Thus center manifold applies to prove the nonlinear stability via asymptotic phase theory. In our case it is equal to the family of stationary solutions. Thus the reduced system on the center manifold vanishes identically. Therefore there is no nonlinearity at all to the zero eigenvalue which corresponds to $l=0$. This is a first hint that the nonlinearity vanishes up to the order we need. 
3.3. Introduction to Renormalization Theory. In this section we explain the use of renormalization theory (cf. [BK92]) in analysing the asymptotic behavior of the perturbations for nonlinear diffusion problems. As an introductory example we consider the above nonlinear diffusion equation

$$
\partial_{t} u=u_{x x}+u^{p},(x \in \mathbb{R}),
$$

with $p>3$. For simplicity assume additionally $p \in N$. Let us introduce again $H^{m}(\mathbb{R}, \mathbb{C})$ as the Sobolev-space for which the derivatives up to order $m$ are in $L^{2}(\mathbb{R}, \mathbb{C})$. A function $u$ lies in $H^{m}(n)$, if $x \mapsto u(x)\left(1+|x|^{2}\right)^{n / 2}$ lies in $H^{m}$. It is well known that Fourier transform is an isomorphism from $H^{m}(n)$ into $H^{n}(m)$. It is a continuous mapping from $L^{1}$ into $L^{\infty}$, but not vice versa.

3.3.1. Linear Diffusive Stability. In Fourier space the diffusion equation $\partial_{t} u=u_{x x}$ is given by $\partial_{t} \hat{u}=-k^{2} \hat{u}$. The solutions are given by $\hat{u}(k, t)=e^{-k^{2} t} \hat{u}(k, 0)$. We have

$$
\begin{aligned}
\|u\|_{H^{m}(n)} & \leqq C\|\hat{u}\|_{H^{n}(m)}=C\left(\int \sum_{j=0}^{n}\left(\partial_{k}^{J} \hat{u}\right)^{2}\left(1+k^{2}\right)^{m}\right)^{1 / 2} \\
& \leqq C\left(1+t^{(2 n-1) / 4}\right)\left\|\left.\hat{u}\right|_{t=0}\right\|_{H^{n}(m)} \leqq C\left(1+t^{(2 n-1) / 4}\right)\left\|\left.u\right|_{t=0}\right\|_{H^{m}(n)} .
\end{aligned}
$$

It is easy to see that these estimates are sharp. Thus $\left(\mathscr{B}, H^{m}(n)\right)$-stability cannot be expected for $n>0$, where $\mathscr{B}$ stands for some Banach space.

Solutions to diffusion equations decay in a characteristic way to zero. This can be observed by rescaling the solutions by $\left(\mathscr{L}_{1 / L} \hat{u}\right)(k)=\hat{u}(k / L)$. tions

Since $|\hat{u}(a)-\hat{u}(a)| \leqq C|a-b|^{\min (1, \max (0, n-1 / 2))}\|\hat{u}\|_{H^{n}}$, we obtain for the solu-

$$
\begin{aligned}
& \left\|\left(\mathscr{L}_{1 / L} \hat{u}\right)\left(k, L^{2}\right)-\left.\hat{u}(0)\right|_{t=0} e^{-k^{2}}\right\|_{H^{n}(m)} \leqq\left\|e^{-k^{2}}\left[\left.\hat{u}(k / L)\right|_{t=0}-\left.\hat{u}(0)\right|_{t=0}\right]\right\|_{H^{n}(m)} \\
& \leqq\left(C / L^{\min (1, \max (0, n-1 / 2))}\right)\left\|\left.\hat{u}\right|_{t=0}\right\|_{H^{n}(m)} .
\end{aligned}
$$

Thus, the optimal decay rate is obtained for $n \geqq 3 / 2$. In $x$-space we have (9) and (1). From (1) follows the $\left(H^{2}(2), L^{\infty}\right)$-stability.

\subsubsection{The Nonlinear Case. Now we consider the nonlinear problem (10).}

Theorem 6. The solution $u \equiv 0$ of $(10)$ is $\left(H^{2}(2), L^{\infty}\right)$-stable. There exists a $\delta>0$ such that for all initial conditions of (10) with $\left\|u_{0}\right\|_{H^{2}(2)}<\delta$ the following holds. There exists an $A^{*} \in \mathbb{R}$ depending on $u_{0}$ such that the solutions $u$ with $\left.u\right|_{t=0}=u_{0}$ behave asymptotically for $t \rightarrow \infty$ as

$$
\left\|\sqrt{t} u(x \sqrt{t}, t)-\sqrt{\pi} A^{*} e^{-x^{2} / 4}\right\|_{H^{2}(2)}=\mathcal{O}\left(t^{-\min (1, p-3) / 2}\right) .
$$

Proof. After the considerations of the last section it remains to show (12). Using the fact that the Fourier transform is an isomorphism in $H^{2}(2)$ we may write (10) as

$$
\partial_{t} \hat{u}=-k^{2} \hat{u}+\hat{u}^{* p},
$$

where $* p$ denotes the $p$-times convolution $(u * v)(k)=\int u(k-l) v(l) d l$. With the scalings $k=K / L^{n}, t=L^{2 n} T$, and $L>1$ we obtain for $v_{n}(K, T)=\hat{u}(k, t)$ the equation

$$
\partial_{T} v_{n}=-K^{2} v_{n}+\left(L^{n(3-p)}\right) v_{n}^{* p},
$$


where we have used

$$
\begin{gathered}
\mathscr{L}_{L} u * \mathscr{L}_{L} v=\frac{1}{L} \mathscr{L}_{L}(u * v) \text { and } \\
\exists C>0 \forall u, v \in H^{n}(m):\|u * v\|_{H^{n}(m)} \leqq C\|u\|_{H^{n}(m)}\|v\|_{H^{n}(m)}
\end{gathered}
$$

if $m>1 / 2$, due to Sobolev's embedding theorem. We denote constants uniformly by $C$ if they can be chosen independent of $L$.

Equivalently to the solution of $(13)$ on the time interval $[1, \infty)$ with initial condition $\left.\hat{u}\right|_{t=1}$ is the following process:

1) $n=1$ : Equation (14) is solved on the time interval $\left[1 / L^{2}, 1\right]$. Then $\mathscr{L}_{1 / L} v_{1}(\cdot, 1)$

is taken as the initial condition for $n=2$, i.e. $v_{2}\left(\cdot, 1 / L^{2}\right)=\mathscr{L}_{1 / L} v_{1}(\cdot, 1)$.

2) $n \rightarrow n+1$ : Equation (14) is solved on the time interval $\left[1 / L^{2}, 1\right]$. Then $\mathscr{L}_{1 / L}$ $v_{n}(\cdot, 1)$ is taken as the initial condition for $n+1$, i.e. $v_{n+1}\left(\cdot, 1 / L^{2}\right)=$ $\mathscr{L}_{1 / L} v_{n}(\cdot, 1)$.

Thus we have

$$
v_{n}(K, T)=e^{-K^{2}\left(T-1 / L^{2}\right)} v_{n-1}(K / L, 1)+L^{n(3-p)} \int_{1 / L^{2}}^{T} e^{-K^{2}(T-s)} v_{n}^{* p}(K, s) d s .
$$

To control the magnitude of the solutions on the time interval $\left[1 / L^{2}, 1\right]$ we need

Lemma 7. Let $\left\|\left.v_{n-1}\right|_{T=1}\right\|_{H^{2}(2)}<\delta$ and $\delta<L^{-\alpha}$ for some $\alpha>5$. Then there exist $C_{1}, L_{0}>0$ such that for all $L>L_{0}$ we have

$$
R:=\sup _{T \in\left[1 / L^{2}, 1\right]}\left\|v_{n}(T)\right\|_{H^{2}(2)} \leqq C_{1} L^{5 / 2-\alpha} .
$$

Proof. We have $\left\|\mathscr{L}_{1 / L} v\right\|_{H^{2}(2)} \leqq C L^{5 / 2}\|v\|_{H^{2}(2)}$. Thus the linear terms in (16) can be estimated by

$$
\begin{aligned}
& \sup _{T \in\left[1 / L^{2}, 1\right]}\left\|K \mapsto e^{-K^{2}\left(T-1 / L^{2}\right)} v_{n-1}(K / L, 1)\right\|_{H^{2}(2)} \\
& \quad \leqq \sup _{T \in\left[1 / L^{2}, 1\right]}\left\|K \mapsto e^{-K^{2}\left(T-1 / L^{2}\right)}\right\|_{C_{b}^{2}}\left\|K \mapsto v_{n-1}(K / L, 1)\right\|_{H^{2}(2)} \leqq C L^{5 / 2} \delta .
\end{aligned}
$$

For the nonlinear terms we obtain

$$
\sup _{T \in\left[1 / L^{2}, 1\right]}\left\|K \mapsto L^{n(3-p)} \int_{1 / L^{2}}^{T} e^{-K^{2}(T-s)} v_{n}^{* p}(K, s) d s\right\|_{H^{2}(2)} \leqq C L^{n(3-p)} R^{p} .
$$

Choosing $\delta$ and $R$ in the above way it is easy to see that the right-hand side of (16) defines a contraction in a ball of radius $C L^{5 / 2-\alpha}$ in $C\left(\left[1 / L^{2}, 1\right], H^{2}(2)\right)$ if $L$ is chosen sufficiently large. Therefore the solution which is the fixed point of this contraction stays in $H^{2}(2)$ in a ball of the same radius $C L^{5 / 2-\alpha}$. Note that the choice of $\alpha$ is not optimal in this case.

Now we show the convergence of the points $\left.v_{n}\right|_{T=1}$ towards $A^{*} e^{-K^{2}}$ in $H^{2}(2)$ for an $A^{*} \in \mathbb{R}$. To do so, let us define the projection $\Pi v_{n}=v_{n}(K=0)$ and $\psi=e^{-K^{2}}$. 
Moreover, we define $\left.v_{n}\right|_{T=1}=A_{n} \psi+\rho_{n}$, with $\rho_{n}(K=0)=0$ and $A_{n} \in \mathbb{R}$. Thus (16) splits up into

$$
\begin{aligned}
A_{n+1}= & A_{n}+\Pi L^{n(3-p)} \int_{1 / L^{2}}^{1} e^{-K^{2}(1-s)} v_{n+1}^{* p}(K, s) d s, \\
\rho_{n+1}(K)= & e^{-K^{2}\left(1-1 / L^{2}\right)} \rho_{n}(K / L)+L^{n(3-p)} \int_{1 / L^{2}}^{1} e^{-K^{2}(1-s)} v_{n+1}^{* p}(K, s) d s \\
& +e^{-K^{2}\left(1-1 / L^{2}\right)} A_{n} \psi(K / L)-A_{n+1} \psi(K) .
\end{aligned}
$$

For the terms on the left-hand side we get the following estimates:

a) $\left\|K \mapsto L^{n(3-p)} \int_{1 / L^{2}}^{1} e^{-K^{2}(1-s)} v_{n+1}^{* p}(K, s) d s\right\|_{H^{2}(2)} \leqq C R^{p} L^{n(3-p)}$,

b) $|\Pi u| \leqq C\|u\|_{H^{2}(2)}$,

c) $\left\|K \mapsto e^{-K^{2}\left(1-1 / L^{2}\right)} \rho_{n}(K / L)\right\|_{H^{2}(2)} \leqq(C / L)\left\|\rho_{n}\right\|_{H^{2}(2)}$ (see (11)),

d) $\left\|K \mapsto e^{-K^{2}\left(1-1 / L^{2}\right)} A_{n} \psi(K / L)-A_{n+1} \psi(K)\right\|_{H^{2}(2)} \leqq C\left|A_{n+1}-A_{n}\right|$.

Using these estimates we obtain

$$
\begin{aligned}
&\left|A_{n+1}-A_{n}\right| \leqq C L^{n(3-p)} R^{p}, \\
&\left\|\rho_{n+1}\right\|_{H^{2}(2)} \leqq(C / L)\left\|\rho_{n}\right\|_{H^{2}(2)}+C L^{n(3-p)} R^{p} .
\end{aligned}
$$

Suppose now that $\sup _{T \in\left[1 / L^{2}, 1\right]} \sup _{n}\left\|v_{n}(T)\right\|_{H^{2}(2)} \leqq R$ and that $L>2 C$. Then it is clear that $\left\|\rho_{n}\right\|_{H^{2}(2)} \rightarrow 0$ for $n \rightarrow \infty$. Since the $A_{n}$ behave as a geometric serie there exists an $A^{*}$ such that $A_{n} \rightarrow A^{*}$.

We are done if we show the existence of a $R>0$ such that $\sup _{T \in\left[1 / L^{2}, 1\right]} \sup _{n} \| v_{n}$ $(T) \|_{H^{2}(2)} \leqq R$. Recall that $\left\|\left.v_{n}\right|_{T=1}\right\|_{H^{2}(2)} \leqq C\left|A_{n}\right|+\left\|\rho_{n}\right\|_{H^{2}(2)}$. Using Lemma 7 it suffices to show that $C\left|A_{n}\right|+\left\|\rho_{n}\right\|_{H^{2}(2)}=: \delta_{n} \leqq 2 \delta_{0}:=2\left(C\left|A_{0}\right|+\left\|\rho_{0}\right\|_{H^{2}(2)}\right)<L^{-\alpha}$ for all $n$ and $\alpha>5$. We obtain $\delta_{n+1} \leqq \delta_{n}+C R^{p} L^{n(3-p)}$ and so $\left|\delta_{n+1}-\delta_{n}\right| \leqq$ $2^{-(n+1)}$ if $C R^{p} L^{(3-p)}<1 / 2$, and if $4 C R^{p} L^{(3-p)}=4 C L^{p(5 / 2-\alpha)} L^{(3-p)} \leqq L^{-\alpha}$. To fulfill these assumptions we have to choose $L>L_{0}$ for a $L_{0}$ sufficiently large. Hence $\delta_{n}<2 \delta_{0}$.

Since convergence goes as $L^{-\min (1, p-3) n}$ and since the convergence holds for all $L \in\left[L_{0}, L_{0}^{2}\right]$ we have established $(12)$ and the $\left(H^{2}(2), L^{\infty}\right)$-stability.

Remark. 8. Theorem 6 holds for all nonlinearities $u^{p}\left(\partial_{x} u\right)^{q}$ as long as $p+2 q>$ 3. In Fourier space we have $\left(\mathscr{L}_{1 / L} u\right)^{* p} *\left(i k \mathscr{L}_{1 / L} v\right)^{* p}=\frac{1}{L^{p+2 q-1}} \mathscr{L}_{1 / L}(u * i K v)$. Thus derivatives in $x$-space or vanishing coefficients in Fourier space give higher powers of $L^{-1}$.

\section{The Nonlinear Stability for the Swift-Hohenberg Equation}

Before we state the exact stability result for the stationary solutions of the Swift-Hohenberg equation in Theorem 15 we need some notations. We introduce the used functional analytic set-up and generalize the concept of so-called modefilters. With these preparations the asymptotic behavior of the perturbations and the 
nonlinear stability result can be established. For notational simplicity let us assume in the following $u_{0}=u_{0}(0,0, \varepsilon)=\varepsilon \tilde{u}_{0}$. In some subsequent remarks we explain how to handle general $u_{0}(\omega, \varphi, \varepsilon)$.

4.1. The Functional Analytic Set-Up. Essential for the proof of Theorem 6 in the last section was the representation of the linear evolution operator by Fourier transform. For our problem we need similarly a representation of the semigroup generated by $\mathscr{M}$ through $e^{\mu_{1}(l) t} \sim e^{-4 l^{2} t}$. Fourier transform has to be generalized (cf. [RS72] Vol. 4 Ch. 16) to obtain such a representation of this semigroup. The eigenfunctions $e^{i k x}$ of usual Fourier transform have to be replaced by the eigenfunctions $w_{l}^{j}(x) e^{i l x}$ of $\mathscr{M}$, with $w_{l}(x)=w_{l}(x+2 \pi), l \in[-1 / 2,1 / 2)$, which were computed before.

Similarly to the fact that Fourier transform is an isomorphism in $L^{2}$ or $H^{2}(2)$ we need estimates for the representation by the eigenfunctions $w_{l}^{j}(x) e^{l l x}$. Our starting point is the following relation:

$$
\begin{aligned}
u(x) & =\int e^{i k x} \hat{u}(k) d k=\sum_{j \in Z} \int_{-1 / 2}^{1 / 2} e^{l(l+j) x} \hat{u}(j+l) d l \\
& =\int_{-1 / 2}^{1 / 2} \sum_{j \in Z} e^{i(l+j) x} \hat{u}(j+l) d l=\int_{-1 / 2}^{1 / 2} e^{i l x} \hat{u}_{l}(x) d l,
\end{aligned}
$$

where $\hat{u}_{l}(x)=\sum_{j \in Z} \hat{u}(j+l) e^{i j x}$. The sum and the integral may be interchanged due to Fubini's theorem for $u \in \mathscr{S}$, where $\mathscr{S}$ is the space of Schwartz-functions, i.e. $\mathscr{S}$ contains functions which are sufficiently smooth and decay sufficiently fast for $|x| \rightarrow \infty$. We have

$$
\begin{aligned}
\int|u(x)|^{2} d x & =2 \pi \int|\hat{u}(k)|^{2} d k=2 \pi \sum_{j \in Z} \int_{-1 / 2}^{1 / 2}|\hat{u}(j+l)|^{2} d l \\
& =2 \pi \int_{-1 / 2}^{1 / 2} \sum_{J \in Z}|\hat{u}(j+l)|^{2} d l=\int_{-1 / 2}^{1 / 2} \int_{0}^{2 \pi}\left|\hat{u}_{l}(x)\right|^{2} d x d l,
\end{aligned}
$$

where we have used that Fourier transform is an isomorphism in $L^{2}$. The same formula with regularity and weights looks like

$$
\begin{aligned}
& \sum_{\tilde{r}=0}^{m} \int\left|\partial_{x}^{\tilde{r}} u(x)\right|^{2}\left(1+|x|^{2}\right)^{n} d x \\
& \quad \leqq C \sum_{r=0}^{n} \int\left|\partial_{k}^{r} \hat{u}(k)\right|^{2}\left(1+|k|^{2}\right)^{m} d k \\
& \quad \leqq C \sum_{r=0}^{n} \sum_{j \in Z} \int_{-1 / 2}^{1 / 2}\left|\partial_{k}^{r} \hat{u}(j+l)\right|^{2}\left(1+|j+l|^{2}\right)^{m} d l \\
& \quad \leqq C \sum_{r=0}^{n} \int_{-1 / 2}^{1 / 2} \sum_{j \in Z}\left|\partial_{k}^{r} \hat{u}(j+l)\right|^{2}\left(1+|j+l|^{2}\right)^{m} d l
\end{aligned}
$$


Diffusive Stability of Solutions of the Swift-Hohenberg Equation

$$
\begin{aligned}
& \leqq C \sum_{r=0}^{n} \sum_{\tilde{r}=0}^{m} \int_{-1 / 2}^{1 / 2} \int_{0}^{2 \pi}\left|\partial_{k}^{r} \partial_{x}^{\tilde{r}} \hat{u}_{l}(x)\right|^{2} d x d l \\
& \leqq C \sum_{\tilde{r}=0}^{m} \int\left|\partial_{x}^{\tilde{r}} u(x)\right|^{2}\left(1+|x|^{2}\right)^{n} d x .
\end{aligned}
$$

Definition 9. Let $m, n \in \mathbb{N}_{0}$. Then, we define the spaces

$$
\mathscr{Z}^{n}(m)=H_{\mathrm{per}}^{n}\left([-1 / 2,1 / 2), H_{\mathrm{per}}^{m}[0,2 \pi)\right) \quad \text { and } \quad \mathscr{L}^{n}(m)=H_{\mathrm{per}}^{n}\left([-1 / 2,1 / 2), l^{2}(m)\right),
$$

where $l^{2}(m)=\left\{u:\left.Z \mapsto \mathbb{C}\left|\|u\|_{l^{2}(m)}=\sum_{k \in Z}\right| u_{k}\right|^{2}\left(1+k^{2}\right)^{m / 2}<\infty\right\}$.

The different representations in (18) and the estimates (19) allow us to define linear mappings between these spaces. Compare also with [RS72] Vol. 4 Ch. 16.

Definition 10. Let $m, n \in \mathbb{N}_{0}$. Then we define linear mappings

$$
\begin{gathered}
\mathscr{F}: H^{m}(n) \rightarrow H^{n}(m):(x \in \mathbb{R} \mapsto u(x)) \mapsto\left(k \mapsto \hat{u}(k)=\frac{1}{2 \pi} \int u(x) e^{-i k x} d x\right), \\
\mathscr{S}_{1}: H^{n}(m) \rightarrow \mathscr{L}^{n}(m):(k \in \mathbb{R} \mapsto \hat{u}(k)) \mapsto\left(l \in[-1 / 2,1 / 2) \mapsto(\hat{u}(l+j))_{j \in Z}\right), \\
\mathscr{S}_{2}: \mathscr{L}^{n}(m) \rightarrow \mathscr{Z}^{n}(m):\left(l \in[-1 / 2,1 / 2) \mapsto(\hat{u}(l+j))_{j \in Z}\right) \\
\mapsto\left(l \in[-1 / 2,1 / 2) \mapsto \hat{u}_{l}(x)=\sum_{j} \hat{u}(l+j) e^{i j x}\right), \\
\mathscr{S}_{3}: \mathscr{Z}^{n}(m) \rightarrow H^{m}(n):\left(l \in[-1 / 2,1 / 2) \mapsto \hat{u}_{l}(x)=\sum_{j} \hat{u}(l+j) e^{i j x}\right) \\
\mapsto\left(x \in \mathbb{R} \mapsto \int_{-1 / 2}^{1 / 2} \hat{u}_{l}(x) e^{i l x} d l\right) .
\end{gathered}
$$

Lemma 11. The mappings $\mathscr{F}, \mathscr{S}_{1}, \mathscr{S}_{2}, \mathscr{S}_{3}$ are isomorphisms. For fixed $m, n$ exists $C=C(m, n)>0$ such that

$$
\begin{aligned}
& \left\|\mathscr{F}_{\operatorname{Lin}\left(H^{m}(n) \rightarrow H^{n}(m)\right)}+\right\| \mathscr{F}^{-1} \|_{\operatorname{Lin}\left(H^{n}(m) \rightarrow H^{m}(n)\right)} \leqq C, \\
& \left\|\mathscr{S}_{1}\right\|_{\operatorname{Lin}\left(H^{n}(m) \rightarrow \mathscr{L}^{n}(m)\right)}+\left\|\mathscr{S}_{1}^{-1}\right\|_{\operatorname{Lin}\left(\mathscr{L}^{n}(m) \rightarrow H^{n}(m)\right)} \leqq C, \\
& \left\|\mathscr{S}_{2}\right\|_{\operatorname{Lin}\left(\mathscr{L}^{n}(m) \rightarrow \mathscr{Z}^{n}(m)\right)}+\left\|\mathscr{S}_{2}^{-1}\right\|_{\operatorname{Lin}\left(\mathscr{Z}^{n}(m) \rightarrow \mathscr{L}^{n}(m)\right)} \leqq C, \\
& \left\|\mathscr{S}_{3}\right\|_{\operatorname{Lin}\left(\mathscr{Z}^{n}(m) \rightarrow H^{m}(n)\right)}+\left\|\mathscr{S}_{3}^{-1}\right\|_{\operatorname{Lin}\left(H^{m}(n) \rightarrow \mathscr{Z}^{n}(m)\right)} \leqq C .
\end{aligned}
$$

Proof. This follows directly from (19) and from $\mathscr{S}_{3} \circ \mathscr{S}_{2} \circ \mathscr{S}_{1} \circ \mathscr{F}=i d$.

We introduce the abbreviation $J=\mathscr{S}_{2} \circ \mathscr{S}_{1} \circ \mathscr{F}: H^{2}(2) \rightarrow \mathscr{Z}^{2}(2)$. For the proof of the asymptotic behavior of the perturbations we work in the space $\mathscr{Z}^{2}(2)$. We denote the operation in $\mathscr{Z}^{2}(2)$ which is associated to the pointwise multiplication - in $H^{2}(2)$ by $*$, i.e. for $u, v \in \mathscr{Z}^{2}(2)$ we define $u * v=J\left(J^{-1} u \cdot J^{-1} v\right)$. We have 
for $l \in[-1 / 2,1 / 2)$

$$
\begin{aligned}
(u * v)_{l}(x) & =\sum_{j} \int_{\mathbb{R}} u(l+j-m) v(m) d m e^{i j x} \\
& =\int_{-1 / 2}^{1 / 2} \sum_{j} \sum_{k} u(l+j-n-k) v(n+k) e^{i(j-k) x} e^{i k x} d n \\
& =\int_{-1 / 2}^{1 / 2} u_{l-n}(x) v_{n}(x) d n .
\end{aligned}
$$

To handle the asymptotic behavior of the solutions we introduce the following renormalization operator $\mathscr{L}_{1 / L}$ and the scaled spaces $\mathscr{Z}_{L}^{n}(m)$.

Definition 12. Let $m, n \in \mathbb{N}$. Then we define the spaces $\mathscr{Z}_{L}^{n}(m)=H_{\mathrm{per}}^{n}([-L / 2, L / 2)$, $\left.H_{\mathrm{per}}^{m}(0,2 \pi)\right)$ and the mappings

$$
\mathscr{L}_{1 / L}:\left\{\begin{array}{l}
\mathscr{Z}^{n}(m) \rightarrow \mathscr{Z}_{L}^{n}(m) \\
\hat{u}_{l}(x) \mapsto \hat{u}_{l / L}(x)
\end{array} .\right.
$$

From the Ginzburg-Landau formalism we know that the attracted neighborhood should be of order $\mathcal{O}(\varepsilon)$ in $L^{\infty}$. Therefore we rescale (3) in such a way that the solutions of the rescaled equation are of order $\mathcal{O}(1)$ for $\varepsilon \rightarrow 0$. Thus we introduce $\tilde{v} \in \mathscr{Z}_{1 / \varepsilon}^{2}(2)$ by $v=J^{-1} \mathscr{L}_{1 / \varepsilon} \tilde{v}$ and consider in the following

$$
\begin{aligned}
\partial_{t} \tilde{v} & =\mathscr{L}_{\varepsilon} J \mathscr{M} J^{-1} \mathscr{L}_{1 / \varepsilon} \tilde{v}+\mathscr{L}_{\varepsilon} J N_{1}\left(J^{-1} \mathscr{L}_{1 / \varepsilon} \tilde{v}\right) \\
& =\Lambda \tilde{v}+N_{2}(\tilde{v}) .
\end{aligned}
$$

We define the nonlinear interaction $*_{L}$ in $\mathscr{Z}_{L}^{n}(m)$ by $\mathscr{L}_{L} v_{n} * \mathscr{L}_{L} w_{n}=(1 / L) \mathscr{L}_{L}$ $\left(v_{n} *_{L} w_{n}\right)$. For notational simplicity we write $*$ instead of $*_{L}$. We have the following rules:

$$
\begin{aligned}
\mathscr{L}_{L} v_{n} * \mathscr{L}_{L} v_{n} & =(1 / L) \mathscr{L}_{L}\left(v_{n} * v_{n}\right), \quad \mathscr{L}_{L} v_{n} * \mathscr{L}_{L} w_{n}=(1 / L) \mathscr{L}_{L}\left(v_{n} * w_{n}\right), \\
u_{0} \mathscr{L}_{L} w_{n} & =\varepsilon \mathscr{L}_{L}\left(\tilde{u}_{0} w_{n}\right), \quad \text { with } \varepsilon \tilde{u}_{0}=u_{0},
\end{aligned}
$$

where the roles of $v_{n}$ and $w_{n}$ can be interchanged.

To control the nonlinear interaction in the spaces $\mathscr{Z}_{L}^{n}(m)$ without loss of powers of $L^{-1}$ we have to introduce weights in the $l$-variable.

Definition 13. We define

$$
\mathscr{Y}_{L}(\beta)=\left\{\left.u \in \mathscr{Z}_{L}^{2}(2)\left|\|u\|_{\mathscr{Y}_{L}(\beta)}^{2}=\sum_{r=0}^{2} \sum_{\tilde{r}=0}^{2} \int_{-L / 2}^{L / 2} \int_{0}^{2 \pi}\right| \partial_{l}^{r} \partial_{x}^{\tilde{r}} u_{l}(x)\right|^{2}\left(1+l^{2}\right)^{\beta} d x d l<\infty\right\} .
$$

Using $\|u * v\|_{L^{2}} \leqq\|u\|_{L^{1}}\|v\|_{L^{2}}$ and $\|u\|_{L^{1}} \leqq C\|u\|_{H^{0}(1 / 2+\delta)}$ for all $\delta>0$ gives the following estimates. There exists a $C>0$ such that for all $L \geqq 1$,

$$
\begin{array}{r}
\left\|u_{0} v\right\| \mathscr{\mathscr { Y }}_{L}(\beta) \\
\|C \varepsilon\| \tilde{u}_{0}\left\|_{H^{2}(0,2 \pi)}\right\| v \|_{\mathscr{Y}_{L}(\beta)} \quad \text { and } \\
\|u * v\|_{\mathscr{O}_{L}\left(\beta_{1}\right)} \leqq(C / L)\|u\|_{\mathscr{Y}_{L}\left(\beta_{1}\right)}\|v\|_{\mathscr{O}_{L}\left(\beta_{2}\right)}
\end{array}
$$


if $\beta_{2}>1 / 2$ and $\beta_{2} \geqq \beta_{1} \geqq 0$. The assertions of Remark 8 are generalized to the space $\mathscr{Y}_{L}(\beta)$ in the following lemma.

Lemma 14. Let $f \in C_{\text {per }}^{2}\left([-1 / 2,1 / 2), C^{2}((0,2 \pi), \mathbb{C})\right)$ with $\|f(l, \cdot)\|_{C^{2}((0,2 \pi), \mathbb{C})} \leqq$ $C|l|^{\gamma}$ for a $\gamma \in[0, \beta]$. Then, there exists a $C>0$ such that for all $L>1$ we have

$$
\left\|\left(\mathscr{L}_{1 / L} f\right) u\right\|_{\mathscr{Y}_{L}(\beta-\gamma)} \leqq C L^{-\gamma}\|f\|_{C_{\mathrm{per}}^{2}\left([-1 / 2,1 / 2), C^{2}((0,2 \pi), \mathbb{C})\right)}\|u\|_{\mathscr{Y}_{L}(\beta)} .
$$

Proof. This follows since $\sup _{l}\left|\frac{l^{\gamma} L^{-\gamma}}{\left(1+l^{2}\right)^{\gamma / 2}}\right|<C L^{-\gamma}$.

\subsection{The Result}

Theorem 15. There exists $\varepsilon_{0}>0$ such that the following holds. Fix $\omega, \varphi$, and $\varepsilon \in\left(0, \varepsilon_{0}\right)$ such that $(21)$ is the system corresponding to an Eckhaus-stable solution $u_{0}(\omega, \varphi, \varepsilon)$. Then for all $p \in(0,1 / 2)$ there exist $\delta, C>0$ such that for all $\tilde{v}_{0} \in \mathscr{Y}_{1 / \varepsilon}(2)$ with $\left\|\tilde{v}_{0}\right\|_{\mathscr{Y}_{1 / \varepsilon}(2)}<\delta$ the following holds. The solutions $\tilde{v}=\tilde{v}(l, x, T) \in$ $\mathscr{Y}_{1 / \varepsilon}(2)$ with $\left.\tilde{v}\right|_{T=1}=\tilde{v}_{0}$ and $T=\varepsilon^{2} t$ behave asymptotically as

$$
\begin{aligned}
\| l & \mapsto \mathscr{L}_{1 / \sqrt{T}} \tilde{v}(\cdot, x, T)-A^{*} e^{-C_{1}(\omega, \varepsilon) l^{2}} f_{1}(l=0)(\omega, \varphi, \varepsilon)[x] \|_{\mathscr{Y}_{\sqrt{T} / \varepsilon}(2)} \\
& \leqq C T^{-1 / 2+p} \quad \text { for } T \rightarrow \infty
\end{aligned}
$$

where $A^{*} \in \mathbb{R}$ is a constant which only depends on the initial conditions. We have the asymptotics $C_{1}(\omega, \varepsilon)=\mathcal{O}(1), \delta=\mathcal{O}(1), C=\mathcal{O}(1)$, and $A^{*}=\mathcal{O}(1)$ for $\varepsilon \rightarrow 0$.

Let us translate this result into physical space. With $A^{*} f_{1}(0) \sqrt{\pi} / \sqrt{C_{1}(\omega, \varepsilon)}=\tilde{A} \partial_{x} \tilde{u}_{0}$ and $\tilde{R}=\mathscr{L}_{1 / \sqrt{T}} \tilde{v}-A^{*} e^{-C_{1}(\omega, \varepsilon) l^{2}} f_{1}(0)$ we obtain in the $L^{\infty}$-norm

$$
\begin{aligned}
v(x, t) & =\int_{-1 / 2}^{1 / 2} \tilde{v}(l / \varepsilon, x, T) e^{i l x} d l \\
& =\int_{-1 / 2}^{1 / 2} A^{*} e^{-C_{1}(\omega, \varepsilon) l^{2} T / \varepsilon^{2}} f_{1}(0) e^{i l x} d l+\mathcal{O}\left(T^{-1 / 2+p}\right) \int_{-1 / 2}^{1 / 2} \tilde{R}(l \sqrt{T} / \varepsilon, x, t) e^{i l x} d l \\
& =\frac{\varepsilon}{\sqrt{T}} \tilde{A} e^{-\varepsilon^{2} x^{2} /\left(4 C_{1}(\omega, \varepsilon) T\right)} \partial_{x} \tilde{u}_{0}+\mathcal{O}\left(\varepsilon T^{-1+p}\right) \\
& =\frac{1}{\sqrt{t}} \tilde{A} e^{-x^{2} /\left(4 C_{1}(\omega, \varepsilon) t\right)} \partial_{x} \tilde{u}_{0}+\mathcal{O}\left(\varepsilon\left(\varepsilon^{2} t\right)^{p-1}\right),
\end{aligned}
$$

where we have used $\tilde{R}(\cdot, \cdot, T) \in \mathscr{Y}_{\sqrt{T} / \varepsilon}(2)$. Thus, we have proved

Corollary 16. In physical space we have the asymptotic behavior

$$
\sup _{x}\left|v(x, t)-\varepsilon \frac{1}{\sqrt{\varepsilon^{2} t}} \tilde{A} e^{-x^{2} /\left(4 C_{1}(\omega, \varepsilon) t\right)} \partial_{x} \tilde{u}_{0}\right| \leqq C \varepsilon\left(\varepsilon^{2} t\right)^{-1+p} \quad \text { for } t \rightarrow \infty .
$$

Therefore, we can conclude

Corollary 17. The fixed point $v \equiv 0$ is $\left(J^{-1} \mathscr{L}_{1 / \varepsilon} \mathscr{Y}_{1 / \varepsilon}(2), L^{\infty}\right)$-stable under the flow of (21). 
Let us describe in physical space the set of initial conditions for which such an asymptotic behavior occurs. Consider (21) and let the perturbations be in $\mathscr{Y}_{1 / \varepsilon}(2)$. Then in Fourier space the Fourier modes are concentrated in neighborhoods of the integers with width $\mathcal{O}(\varepsilon)$. In physical space this means that the solutions of $(2)$ can be written as $\varepsilon \tilde{u}_{0}+\sum_{n} \varepsilon A_{n}(\varepsilon x) e^{i n x}$, where $\left\|A_{n}\right\|_{H^{2}(2)} \leqq C \delta=\mathcal{O}(1)$. Therefore the attracted neighborhood is of the same order as the solution in powers of $\varepsilon$ in $L^{\infty}$. The fact $A_{n} \in H^{\beta}$ comes from the weight in $\mathscr{Y}_{1 / \varepsilon}(\beta)$. The following corollary holds.

Corollary 18. There exist $\varepsilon_{0}, \delta>0$ such that the following holds. Let $V_{\delta}=$ $\left\{A \in H^{2}(2) \mid\|A\|_{H^{2}(2)}<\delta\right\}$ be a neighborhood $A_{0}+V_{\delta}$ of the equilibrium $A_{0}$ of the Ginzburg-Landau equation (7). Then for all $\varepsilon \in\left(0, \varepsilon_{0}\right)$ the assertions of Theorem 15 hold for all initial conditions in the set $\mathscr{L}_{\varepsilon} J \tilde{\psi}_{\varepsilon}\left(V_{\delta}\right) \subset \mathscr{Y}_{1 / \varepsilon}(2)$.

Note that the renormalization process for the equilibria of the Ginzburg-Landau equation can be made in the space $H^{2}(2)$.

Using the attractivity result of [Eck93, Sch94c, Sch95] we know that after a time $\mathcal{O}\left(1 / \varepsilon^{2}\right)$ the solutions can be written as

$$
\varepsilon \tilde{u}_{0}+\varepsilon A_{1}(\varepsilon x) e^{i x}+\text { c.c. }+\mathcal{O}\left(\varepsilon^{2}\right)
$$

where again $\left\|A_{1}\right\|_{H^{2}(2)}=\mathcal{O}(1)$. The function $A_{1}$ behaves approximately as a solution of the Ginzburg-Landau equation. Using the diffusive stability result [BK92] for the Ginzburg-Landau equation gives formally with $X=\varepsilon x$ and $T=\varepsilon^{2} t$ that

$$
\begin{aligned}
& \| \varepsilon A_{1}(X, T) e^{i x}+\text { c.c. }+\mathcal{O}\left(\varepsilon^{2}\right)-\left[\varepsilon(T)^{-1 / 2} \tilde{A} e^{-X^{2} /\left(4 C_{1}(\omega, 0) T\right)} e^{i x}+c . c .\right] \|_{L^{\infty}} \\
& \quad \leqq \mathcal{E O}\left(T^{-1}\right)+\mathcal{O}\left(\varepsilon^{2}\right) .
\end{aligned}
$$

Since the solutions of the original system can only be approximated via the solutions of the Ginzburg-Landau equation for $t \leqq \mathcal{O}\left(1 / \varepsilon^{2}\right)$ ([CE90b, vH91, Sch94b]) nothing can be concluded from this, but it fills our result with more life.

Remark. 19. The main point of the proof is to show that the nonlinear terms are really irrelevant. The most difficult terms $s_{1}$ and $s_{2}$ are estimated in the proof of Lemma 24. For their estimates we need Lemma 14 and Lemma 4. To estimate these terms we have to choose $p>0$. This is explained in Remark 26.

Remark. 20. To handle simultaneously the asymptotic behavior of the perturbations and the stability question, the usual definition of stability is generalized. This generalization is natural for problems on the infinite line. This is because on the infinite line there are function spaces which are essentially different. For instance, $C_{b}^{0}([0,1], \mathbb{R}) \subset L^{2}([0,1], \mathbb{R})$, but neither $C_{b}^{0}(\mathbb{R}, \mathbb{R}) \subset L^{2}(\mathbb{R}, \mathbb{R})$, nor $L^{2}(\mathbb{R}, \mathbb{R}) \subset C_{b}^{0}(\mathbb{R}, \mathbb{R})$.

4.3. The Renormalization Process. For notational simplicity we demonstrate first Theorem 15 for $u_{0}=u_{0}(0,0, \varepsilon)$. A combination of Remarks 5 and 27 demonstrates the result for all Eckhaus-stable solutions $u_{0}=u_{0}(\omega, \varphi, \varepsilon)$. In [Sch94b] mode filters are defined which extract the critical, i.e. in [Sch94b] the exponentially increasing, Fourier modes from a function $u$. Here we generalize these concepts of mode filters to extract the diffusive part from a solution. To do so we need the following lemma. 
Lemma 21. Let $\tilde{M} \in C_{\text {per }}^{n}\left([-1 / 2,1 / 2), \operatorname{Lin}\left(H_{\text {per }}^{m_{1}}, H_{\text {per }}^{m_{2}}\right)\right)$. Then $(l \mapsto u(l)) \mapsto(l \mapsto$ $\tilde{M}(l / L) u(l))$ defines a linear mapping from $\mathscr{Z}_{L}^{n}\left(m_{1}\right)$ into $\mathscr{Z}_{L}^{n}\left(m_{2}\right)$. There exists a $C>0$ such that for all $L \geqq 1$ we have the estimate

$$
\|M u\|_{\mathscr{Z}_{L}^{n}\left(m_{2}\right)} \leqq C\left\|\mathscr{L}_{1 / L} \tilde{M}\right\|_{C_{\mathrm{per}}^{n}[-L / 2, L / 2), \operatorname{Lin}\left(H_{\mathrm{per}}^{m_{1}}, H_{\mathrm{per}}^{m_{2}}\right)}\|u\|_{\mathscr{Z}_{L}^{n}\left(m_{1}\right)} .
$$

Proof. This follows directly from the definition of $\mathscr{Z}_{L}^{n}(m)$.

From the computations in Sect. 2 it is clear that there exists an $l_{0}>0$ such that at least in a neighborhood $\left(-\varepsilon l_{0}, \varepsilon l_{0}\right) \subset(-1 / 2,1 / 2)$ for fixed $l$ the largest eigenvalue $\mu_{1}(l)$ is bounded away from all others. So for fixed $l$ through the Dunford-integral

$$
P_{1}(l)=\frac{1}{2 \pi i} \int_{\Gamma}\left(\mathscr{M}_{l}-s\right)^{-1} d s
$$

a projection on the eigenspace $\operatorname{span}\left\{f_{1}(l)\right\}$ is defined, where $\Gamma$ is a curve in the complex plane surrounding $\mu_{1}(l)$ in the resolvent set. Since (5) is a self-adjoint problem in $L_{\text {per }}^{2}(0,2 \pi)$ the projection $P_{1}$ is orthogonal in $L_{\text {per }}^{2}(0,2 \pi)$ for fixed $l$.

Next we define a smooth cut-off function $\chi \in C_{0}^{\infty}$ by

$$
\chi(l)= \begin{cases}0 & |l| \geqq l_{0} / 2 \\ \in[0,1] & l_{0} / 4 \leqq|l| \leqq l_{0} / 2 . \\ 1 & |l| \leqq l_{0} / 4\end{cases}
$$

There exist $C, \varepsilon_{0}>0$ such that for all $\varepsilon \in\left(0, \varepsilon_{0}\right)$ the operator $\tilde{E}_{c}\left(\varepsilon^{2}, l\right)=\chi(l / \varepsilon)$ $P_{1}\left(\varepsilon^{2}, l\right): H^{m} \rightarrow H^{m}$ fulfills $\left\|\mathscr{L}_{\varepsilon} \tilde{E}_{C}\right\|_{C_{\mathrm{per}}^{n}\left[(-1 /(2 \varepsilon), 1 /(2 \varepsilon)), \operatorname{Lin}\left(H_{\mathrm{per}}^{m}, H_{\mathrm{per}}^{m}\right)\right)}<C<\infty$. This holds, because of the representation of $P_{1}(l)$ by the Dunford-integral and because of the orthogonal property.

Definition 22. According to Lemma 21 the multiplier $\tilde{E}_{c}$ defines the so-called mode filter $E_{c}: \mathscr{Z}^{m}(n) \rightarrow \mathscr{Z}^{m}(n)$. We define linear mappings $\tilde{e}_{c}(l): H_{\mathrm{per}}^{m} \rightarrow \mathbb{C}$ by $\left(\tilde{e}_{c}(l) u\right) f_{1}(l)=\tilde{E}_{c}(l) u$. Moreover, we define $E_{s}=i d$. $-E_{c}$. Since $E_{c}$ and $E_{s}$ are no projections we define auxiliary mode filters $E_{c}^{h}$ and $E_{s}^{h}$ by $\tilde{E}_{c}^{h}(l)=P_{1}(l) \chi(2 l / \varepsilon)$ and $\tilde{E}_{s}^{h}(l)=\left(I d .-P_{1}(l / \varepsilon)\right) \chi(l / 2)$.

An application of Lemma 21 gives the following estimates.

Lemma 23. There exists $C=C(m, n)>0$ independent of $\varepsilon \in\left(0, \varepsilon_{0}\right)$ such that for all $L>1 / \varepsilon$ we have $\left\|\mathscr{L}_{1 / L} E_{c} \mathscr{L}_{L} u\right\|_{\mathscr{O}_{L}(\beta)} \leqq C\|u\|_{\mathscr{O}_{L}(\beta)}$ and $\left\|\mathscr{L}_{1 / L} E_{S} \mathscr{L}_{L} u\right\|_{\mathscr{O}_{L}(\beta)} \leqq$ $C\|u\|_{\mathscr{S}_{L}(\beta)}$. Moreover $E_{c}^{h} E_{c}=E_{c}$, and $E_{s}^{h} E_{s}=E_{s}$.

With these preparations we define the diffusive part $u_{c}$ and the linear exponentially damped part $\tilde{u}_{s}$ to be the solutions of

$$
\begin{aligned}
& \partial_{t} u_{c}=\Lambda u_{c}+\mathscr{L}_{\varepsilon} E_{c} \mathscr{L}_{1 / \varepsilon} N_{2}\left(u_{c}+\tilde{u}_{s}\right), \\
& \partial_{t} \tilde{u}_{s}=\Lambda \tilde{u}_{s}+\mathscr{L}_{\varepsilon} E_{s} \mathscr{L}_{1 / \varepsilon} N_{2}\left(u_{c}+\tilde{u}_{s}\right),
\end{aligned}
$$

where $u_{c}$ and $\tilde{u}_{s}$ should additionally fulfill $u_{c}=\mathscr{L}_{\varepsilon} E_{c}^{h} \mathscr{L}_{1 / \varepsilon} u_{c}$ and $\tilde{u}_{s}=\mathscr{L}_{\varepsilon} E_{s}^{h} \mathscr{L}_{1 / \varepsilon} \tilde{u}_{s}$. The initial conditions are given by $u_{c}=\left.\mathscr{L}_{\varepsilon} E_{c} \mathscr{L}_{1 / \varepsilon} \tilde{v}\right|_{t=0}$ and by $u_{s}=\left.\mathscr{L}_{\varepsilon} E_{s} \mathscr{L}_{1 / \varepsilon} \tilde{v}\right|_{t=0}$. Note that the separation of (21) into (27) is not unique. But if we have found solutions $u_{c}$ and $\tilde{u}_{s}$ of (27) we also have found a solution of (21) since the sum 
of the two equations in (27) equals (21). Since the solutions of (2) are unique it suffices to consider one system (27) to show the assertions of Theorem 15.

We modify this system by introducing coordinates

$$
\tilde{u}_{s}=\Lambda^{-1} \mathscr{L}_{\varepsilon} E_{s} \mathscr{L}_{1 / \varepsilon}\left(3 u_{0} u_{c} * u_{c}\right)+u_{s} .
$$

Note that $\Lambda^{-1}$ exists for the considered values of $l$ and is of order $\mathcal{O}\left(\varepsilon^{-2}\right)$. This transform simplifies the following calculations. Under this transform (27) becomes

$$
\begin{gathered}
\partial_{t} u_{c}=\Lambda u_{c}+N_{3}\left(u_{c}, u_{s}\right), \\
\partial_{t} u_{s}=\Lambda u_{s}+N_{4}\left(u_{c}, u_{s}\right),
\end{gathered}
$$

where

$$
\begin{aligned}
N_{3}\left(u_{c}, u_{s}\right)= & \mathscr{L}_{\varepsilon} E_{c} \mathscr{L}_{1 / \varepsilon} N_{2}\left(u_{c}+\Lambda^{-1} \mathscr{L}_{\varepsilon} E_{s} \mathscr{L}_{1 / \varepsilon}\left(3 u_{0} u_{c} * u_{c}\right)+u_{s}\right) \\
N_{4}\left(u_{c}, u_{s}\right)= & \mathscr{L}_{\varepsilon} E_{s} \mathscr{L}_{1 / \varepsilon} N_{2}\left(u_{c}+\Lambda^{-1} \mathscr{L}_{\varepsilon} E_{s} \mathscr{L}_{1 / \varepsilon}\left(3 u_{0} u_{c} * u_{c}\right)+u_{s}\right) \\
& -\partial_{t}\left[\Lambda^{-1} \mathscr{L}_{\varepsilon} E_{s} \mathscr{L}_{1 / \varepsilon}\left(3 u_{0} u_{c} * u_{c}\right)\right] .
\end{aligned}
$$

We start the renormalization process with noting that the dynamics happens on the Ginzburg-Landau time scale. Thus, we scale the diffusive part $u_{c}$ as

$$
v_{n}(K, x, T)=u_{c}\left(K / L^{n}, x, L^{2 n} T / \varepsilon^{2}\right) .
$$

The scaling of the exponentially damped part comes from the following considerations. By the above coordinate transform the quadratic terms in $u_{c}$ are eliminated. Thus, if $\left.u_{c}\right|_{t=L^{2}}$ has a decay rate $1 / L^{n}$, then $\left.u_{s}\right|_{t=L^{2}}$ has a decay rate $1 / L^{3 n}$ for $n \rightarrow \infty$. Therefore we scale

$$
w_{n}(K, x, T)=L^{2 n(1-p)} u_{s}\left(K / L^{n}, x, L^{2 n} T / \varepsilon^{2}\right) \text { with } p \in(0,1 / 2) .
$$

This scaling seems not to be optimal $(p=0)$, but it is, and it has the advantage that in this limit the variables $w_{n}$ converge towards 0 . Under these scalings the variation of constant formula for (29) is written as

$$
\begin{aligned}
& v_{n}(T)=e^{L^{2 n} \varepsilon^{-2} \Lambda_{n}^{c}\left(T-1 / L^{2}\right)} v_{n-1}(K / L, 1) \\
& +\varepsilon^{-2} L^{2 n} \int_{1 / L^{2}}^{T} e^{L^{2 n} \varepsilon^{-2} \Lambda_{n}^{c}(T-\tau)} N_{c}\left(v_{n}(\tau), w_{n}(\tau)\right) d \tau, \\
& w_{n}(T)=e^{L^{2 n} \varepsilon^{-2} \Lambda_{n}^{s}\left(T-1 / L^{2}\right)} w_{n-1}(K / L, 1) \\
& +\varepsilon^{-2} L^{2 n(2-p)} \int_{1 / L^{2}}^{T} e^{L^{2 n} \varepsilon^{-2} \Lambda_{n}^{s}(T-\tau)} N_{s}\left(v_{n}(\tau), w_{n}(\tau)\right) d \tau,
\end{aligned}
$$

with

$$
\begin{aligned}
\Lambda_{n}^{c} & =\mathscr{L}_{\varepsilon / L^{n}} E_{c}^{h} \mathscr{L}_{1 / \varepsilon} \Lambda \mathscr{L}_{L^{n}}, \quad \Lambda_{n}^{s}=\mathscr{L}_{\varepsilon / L^{n}} E_{c}^{h} \mathscr{L}_{1 / \varepsilon} \Lambda \mathscr{L}_{L^{n}}, \\
N_{c}\left(v_{n}, w_{n}\right) & =\mathscr{L}_{1 / L^{n}} N_{3}\left(v_{n}, L^{2 n(p-1)} w_{n}\right), \quad N_{s}\left(v_{n}, w_{n}\right)=\mathscr{L}_{1 / L^{n} N_{4}\left(v_{n}, L^{2 n(p-1)} w_{n}\right) .}
\end{aligned}
$$


We proceed as above. Equivalently to the solution of (29) on the time interval $\left[1 / \varepsilon^{2}, \infty\right)$ with initial conditions $\left.\left(u_{c}, u_{s}\right)\right|_{t=1 / \varepsilon^{2}}$ is the following process:

1) $n=1$ : Equation (30) is solved on the time interval $\left[1 / L^{2}, 1\right]$. Then $\left(\mathscr{L}_{1 / L} v_{1}\right)$ $\left.(\cdot, x, 1), \mathscr{L}_{1 / L} w_{1}(\cdot, x, 1)\right)$ is taken as the initial condition for $n=2$, i.e. $v_{2}\left(\cdot, x, 1 / L^{2}\right)$ $=\mathscr{L}_{1 / L} v_{1}(\cdot, x, 1)$ and $w_{2}\left(\cdot, x, 1 / L^{2}\right)=\mathscr{L}_{1 / L} w_{1}(\cdot, x, 1)$.

2) $n \rightarrow n+1$ : Equation (30) is solved on the time interval $\left[1 / L^{2}, 1\right]$. Then $\left(\mathscr{L}_{1 / L} v_{n}(\cdot, x, 1), \mathscr{L}_{1 / L} w_{n}(\cdot, x, 1)\right)$ is taken as the initial condition for $n+1$, i.e. $v_{n+1}\left(\cdot, x, 1 / L^{2}\right)=\mathscr{L}_{1 / L} v_{n}(\cdot, x, 1)$ and $w_{n+1}\left(\cdot, x, 1 / L^{2}\right)=\mathscr{L}_{1 / L} w_{n}(\cdot, x, 1)$.

To control the magnitude of the solutions on the time interval $\left[1 / L^{2}, 1\right]$ we need

Lemma 24. Let $\left\|\left.v_{n-1}\right|_{T=1}\right\|_{\mathscr{Y}_{L^{n-1 / \varepsilon}}(2)}+\left\|\left.w_{n-1}\right|_{T=1}\right\|_{\mathscr{Y}_{L^{n-1 / \varepsilon}}(2)}<\delta$ and $\delta<L^{-\alpha}$ for some $\alpha>5$. Then there exist $C_{2}, L_{0}>0$ such that for all $L>L_{0}$ we have

$$
R:=\sup _{T \in\left[1 / L^{2}, 1\right]}\left\|v_{n}(T)\right\| \mathscr{Y}_{L^{n} / \varepsilon}(2)+\sup _{T \in\left[1 / L^{2}, 1\right]}\left\|w_{n}(T)\right\|_{\mathscr{Y}_{L^{n} / \varepsilon}(2)} \leqq C_{2} L^{5 / 2-\alpha} .
$$

Proof. See Sect. 4.4 .

In the following we denote constants uniformly by $C$ if they can be chosen independent of $L$ and $\varepsilon$. We show now the convergence of the points $\left.v_{n}\right|_{T=1}$ towards $A^{*} e^{-C_{1}(\omega, \varepsilon) l^{2}} f_{1}(l=0)$ for an $A^{*} \in \mathbb{R}$ and of $\left.w_{n}\right|_{T=1}$ towards 0 . To do so, let us define $\psi=e^{-C_{1}(\omega, \varepsilon) l^{2}} f_{1}(l=0)$ and the projection $\Pi v_{n}=v_{n}(K=0)$. Moreover, we define $\left.w_{n}\right|_{T=1}=\phi_{n}$ and $\left.v_{n}\right|_{T=1}=A_{n} \psi+\rho_{n}$, with $\rho_{n}(K=0)=0$ and $A_{n} \in \mathbb{R}$. Thus (30) splits up into

$$
\begin{aligned}
& A_{n}=A_{n-1}+\Pi \varepsilon^{-2} L^{2 n} \int_{1 / L^{2}}^{1} e^{L^{2 n} \varepsilon^{-2} \Lambda_{n}^{c}(1-\tau)} N_{c}\left(v_{n}, w_{n}\right) d \tau, \\
& \rho_{n}(K)=e^{L^{2 n} \varepsilon^{-2} \Lambda_{n}^{c}\left(1-1 / L^{2}\right)} \rho_{n-1}(K / L)+\varepsilon^{-2} L^{2 n} \int_{1 / L^{2}}^{1} e^{L^{2 n} \varepsilon^{-2} \Lambda_{n}^{c}(1-\tau)} N_{c}\left(v_{n}, w_{n}\right) d \tau \\
& +e^{L^{2 n} \varepsilon^{-2} \Lambda_{n}^{c}\left(1-1 / L^{2}\right)} A_{n-1} \psi(K / L)-A_{n} \psi(K), \\
& \phi_{n}(K)=e^{L^{2 n} \varepsilon^{-2} \Lambda_{n}^{s}\left(1-1 / L^{2}\right)} \phi_{n-1}(K / L)+\varepsilon^{-2} L^{2 n(2-p)} \int_{1 / L^{2}}^{1} e^{L^{2 n} \varepsilon^{-2} \Lambda_{n}^{s}(1-\tau)} N_{s}\left(v_{n}, w_{n}\right) d \tau
\end{aligned}
$$

Note that the initial conditions $A_{0} \in \mathbb{R}, \rho_{0} \in \mathscr{Y}_{1 / \varepsilon}$, and $\phi_{0} \in \mathscr{Y}_{1 / \varepsilon}$ are of order $\mathcal{O}(1)$ for $\varepsilon \rightarrow 0$. For the terms on the left-hand side we get the following estimates.

Lemma 25. Suppose $\sup _{T \in\left[1 / L^{2}, 1\right]}\left\|v_{n}(T)\right\|_{\mathscr{Y}_{L^{n} / \varepsilon}(2)}+\sup _{T \in\left[1 / L^{2}, 1\right]}\left\|w_{n}(T)\right\|_{\mathscr{Y}_{L^{n} / \varepsilon}(2)} \leqq R$. Then for all $p \in(0,1 / 2)$ there exist $L_{0}, \varepsilon_{0}, C>0$, such that for all $\varepsilon \in\left(0, \varepsilon_{0}\right)$ and all $L>L_{0}$ :

a) $\left\|\varepsilon^{-2} L^{2 n} \int_{1 / L^{2}}^{1} e^{L^{2 n} \varepsilon^{-2} \Lambda_{n}^{c}(1-\tau)} N_{c}\left(v_{n}, w_{n}\right) d \tau\right\|_{\mathscr{Y}_{L^{n} / \varepsilon}(2)} \leqq C R^{2} L^{n(2 p-1)}$,

b) $\left\|\varepsilon^{-2} L^{2 n(2-p)} \int_{1 / L^{2}}^{1} e^{L^{2 n} \varepsilon^{-2} \Lambda_{n}^{s}(1-\tau)} N_{s}\left(v_{n}, w_{n}\right) d \tau\right\|_{\mathscr{Y}_{L^{n} / \varepsilon}(2)} \leqq C R^{2} L^{-2 n p}$, 
c) $|\Pi u| \leqq C\|u\|_{\mathscr{Y}_{L^{n} / \varepsilon}(2)}$,

d) $\left\|K \mapsto e^{L^{2 n} \varepsilon^{-2} \Lambda_{n}^{c}\left(1-1 / L^{2}\right)} \rho_{n-1}(K / L)\right\|_{\mathscr{Y}_{L^{n} / \varepsilon}(2)} \leqq(C / L)\left\|\rho_{n-1}\right\|_{\mathscr{Y}_{L^{n-1 / \varepsilon}}(2)}$,

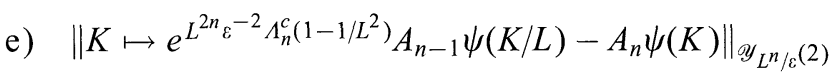

$$
\leqq C\left|A_{n}-A_{n-1}\right|+C R L^{-n},
$$

f) $\left\|K \mapsto e^{L^{2 n} \varepsilon^{-2} \Lambda_{n}^{s}\left(1-1 / L^{2}\right)} \phi_{n-1}(K / L)\right\|_{\mathscr{Y}_{L^{n} / \varepsilon}(2)} \leqq C e^{-C L^{2 n}}\left\|\phi_{n-1}\right\|_{\mathscr{Y}_{L^{n-1 / \varepsilon}}(2)}$.

\section{Proof. See Sect. 4.4.}

Using these estimates we obtain

$$
\begin{aligned}
\left|A_{n}-A_{n-1}\right| & \leqq C L^{n(2 p-1)} R^{2}, \\
\left\|\rho_{n}\right\|_{\mathscr{Y}_{L^{n} / \varepsilon}(2)} & \leqq(C / L)\left\|\rho_{n-1}\right\|_{\mathscr{\partial} L_{L^{n-1 / \varepsilon}}(2)}+C L^{n(2 p-1)} R^{2}+C L^{-n} R, \\
\left\|\phi_{n}\right\|_{\mathscr{Y}_{L^{n} / \varepsilon}(2)} & \leqq C e^{-C L^{2 n}\left\|\phi_{n-1}\right\|_{\mathscr{Y}_{L^{n-1 / \varepsilon}}(2)}+C L^{-2 n p} R^{2} .}
\end{aligned}
$$

Suppose now that $\sup _{T \in\left[1 / L^{2}, 1\right]} \sup _{n}\left(\left\|w_{n}(T)\right\|_{\mathscr{Y}_{L^{n} / \varepsilon}(2)}+\left\|v_{n}(T)\right\|_{\mathscr{Y}_{L^{n} / \varepsilon}(2)}\right) \leqq R$ and choose $L$ so large that $C e^{-C L^{2}}<1 / 2$ and $L>2 C$. Then it is clear that $\left\|\phi_{n}\right\|_{\mathscr{Y}_{L^{n} / \varepsilon}(2)}+$ $\left\|\rho_{n}\right\|_{\mathscr{Y}_{L^{n} / \varepsilon}(2)} \rightarrow 0$ for $n \rightarrow \infty$. Moreover, there exists an $A^{*}$ such that $A_{n} \rightarrow A^{*}$.

Then we have

$$
\begin{aligned}
\left\|\mathscr{L}_{1 / L^{n}} \tilde{v}-A^{*} \psi\right\|_{\mathscr{Y}_{L^{n} / \varepsilon}(2)} & \leqq C\left(\left|A^{*}-A_{n}\right|+\left\|\rho_{n}\right\|_{\mathscr{Y}_{L^{n} / \varepsilon}(2)}+L^{2 n(p-1)}\left\|\phi_{n}\right\|_{\mathscr{Y}_{L^{n} / \varepsilon}(2)}\right) \\
& \leqq C L^{n(2 p-1)} .
\end{aligned}
$$

Since this discrete convergence holds for all $L \in\left[L_{0}, L_{0}^{2}\right]$ we are done if we show the existence of a $R>0$ such that $\sup _{T \in\left[1 / L^{2}, 1\right]} \sup _{n}\left(\left\|w_{n}(T)\right\|_{\mathscr{Y}_{L^{n} / \varepsilon}(2)}+\left\|v_{n}(T)\right\|_{\mathscr{Y}_{L^{n} / \varepsilon}(2)}\right) \leqq$ $R$. Recall that $\left\|\left.v_{n}\right|_{T=1}\right\|_{\mathscr{Y}_{L^{n} / \varepsilon}(2)} \leqq C\left|A_{n}\right|+\left\|\rho_{n}\right\|_{\mathscr{Y}_{L^{n} / \varepsilon}(2)}$. Let us define

$$
\delta_{n}:=C\left|A_{n}\right|+\left\|\rho_{n}\right\|_{\mathscr{Y}_{L^{n} / \varepsilon}(2)}+\left\|\phi_{n}\right\|_{\mathscr{Y}_{L^{n} / \varepsilon}(2)} .
$$

Choose now $\delta_{0}<L^{-\alpha_{0}}$ with $\alpha_{0}=7+\gamma$ for a $\gamma>0$. Using Lemma 24 shows that $R_{1} \leqq L^{-\alpha_{0}+5 / 2}$, where $R_{n}:=\sup _{T \in\left[1 / L^{2}, 1\right]}\left(\left\|v_{n}(T)\right\|_{\mathscr{Y}_{L^{n} / \varepsilon}(2)}+\left\|w_{n}(T)\right\|_{\mathscr{Y}_{L^{n} / \varepsilon}(2)}\right)$. Thus

$$
\begin{aligned}
\delta_{1} & =\delta_{0}+C L^{2 p-1} R_{1}^{2}+C L^{-1} R_{1}+C L^{-2 p} R_{1}^{2} \\
& \leqq C L^{-1} R_{1} \leqq C L^{\alpha_{1}}
\end{aligned}
$$

with $\alpha_{1}=\alpha_{0}+3 / 2=11 / 2+\gamma$. Again with Lemma 24 we obtain $R_{2}=L^{-\alpha_{1}+5 / 2}$ and so $\delta_{2} \leqq C L^{-2} R_{2} \leqq C L^{\alpha_{2}}$ with $\alpha_{2}=\alpha_{1}+1 / 2=5+\gamma$.

Now we can proceed as in the example above. Using again Lemma 24 it suffices to show $\delta_{n} \leqq 2 \delta_{2}$ for all $n \geqq 3$. This gives as before the conditions

$$
\begin{aligned}
& C L^{n(2 p-1)} R_{n}^{2}+C L^{-n} R_{n}+C L^{-2 n p} R_{n}^{2} \\
& \quad=C L^{n(2 p-1)} L^{2\left(-\alpha_{2}+5 / 2\right)}+C L^{-n} L^{-\alpha_{2}+5 / 2}+C L^{-2 n p} L^{2\left(-\alpha_{2}+5 / 2\right)} \leqq\left(L^{-\alpha_{2}}\right) / 4
\end{aligned}
$$


and $L^{\min (p, 2 p-1)}<1 / 2$. They are fulfilled for $L>L_{0}$ for a $L_{0}>1$ sufficiently large independent of $\varepsilon$ since $\alpha_{2}>5$ and since $n \geqq 3$. Note that this point gives not the restriction $p \neq 0$. For $p=0$ the variable $\phi_{n}$ has a different limit and the rate of convergence is again $L^{-n}$.

\subsection{The Proofs}

Proof of Lemma 24. Without loss of generality we can assume

$$
\sup _{T \in\left[1 / L^{2}, 1\right]}\left(\left\|v_{n}(T)\right\|_{\mathscr{Y}_{L^{n} / \varepsilon}(2)}+\left\|w_{n}(T)\right\|_{\mathscr{Y}_{L^{n} / \varepsilon}(2)}\right) \leqq 1
$$

a) We start with estimating the linear terms. Let $g \in \mathscr{Y}_{L^{n-1 / \varepsilon}}(2)$. Then

$$
\left\|e^{L^{2 n} \varepsilon^{-2} \Lambda_{n}\left(T-1 / L^{2}\right)} \mathscr{L}_{1 / L} g\right\|_{\mathscr{Y}_{L^{n} / \varepsilon}(2)} \leqq C\left\|\mathscr{L}_{1 / L} g\right\|_{\mathscr{Y}_{L^{n} / \varepsilon}(2)} \leqq C L^{5 / 2}\|g\|_{\mathscr{Y}_{L^{n-1 / \varepsilon}}(2)},
$$

and so

$$
\sup _{T \in\left[1 / L^{2}, 1\right]}\left\|K \mapsto e^{L^{2 n} \varepsilon^{-2} \Lambda_{n}\left(T-1 / L^{2}\right)}\left(v_{n-1}(K / L, 1), w_{n-1}(K / L, 1)\right)\right\|_{\mathscr{Y}_{L^{n} / \varepsilon}(2)} \leqq C L^{5 / 2} \delta .
$$

b) Let $E_{\alpha n}^{h}=\mathscr{L}_{\varepsilon / L^{n}} E_{\alpha}^{h} \mathscr{L}_{L^{n} / \varepsilon}$ for $\alpha=c, s$. To estimate the nonlinear terms in the equations for $w_{n}$ we note that

$$
\left\|e^{L^{2 n} \varepsilon^{-2} \Lambda_{n}^{S}\left(T-1 / L^{2}\right)} E_{s n}^{h} g\right\|_{\mathscr{Y}_{L^{n} / \varepsilon}(2)} \leqq e^{-C L^{2 n} T}\|g\|_{C\left(\left[1 / L^{2}, 1\right], \mathscr{Y}_{L^{n} / \varepsilon}(2)\right)},
$$

since the spectrum of $\Lambda_{n}^{s}$ is below $-C \varepsilon^{2}$. Using the rules (22) and (23) shows with $L^{2 n} / \varepsilon$ instead of $L$ that

$$
\left\|N_{S}\right\|_{\mathscr{Y}_{L^{n} / \varepsilon}(2)} \leqq \frac{C \varepsilon^{2}}{L^{2 n}}\left(\left\|v_{n}\right\|_{\mathscr{Y}_{L^{n} / \varepsilon}(2)}+\left\|w_{n}\right\|_{\mathscr{Y}_{L^{n} / \varepsilon}(2)}\right)^{2}
$$

and so

$$
\begin{aligned}
& \sup _{T \in\left[1 / L^{2}, 1\right]} \| \varepsilon^{-2} L^{2 n(2-p)} \int_{1 / L^{2}}^{T} e^{L^{2 n} \varepsilon^{-2} \Lambda_{n}^{s}(T-\tau)} N_{s} d \tau \\
& \leqq C R^{2} L^{2 n(1-p)} \int_{1 / L^{2}}^{1} e^{-C L^{2 n} T} d T \leqq C L^{-2 n p} R^{2} .
\end{aligned}
$$

c) More complicated are the estimates for the nonlinear terms in the equations for $v_{n}$. The linear semigroup for the diffusive part fulfills

$$
\left\|e^{L^{2 n} \varepsilon^{-2} \Lambda_{n}^{c}\left(T-1 / L^{2}\right)} E_{c n}^{h} g\right\|_{\mathscr{Y}_{L^{n} / \varepsilon}(2)} \leqq C\left(T-1 / L^{2}\right)^{\beta / 2-1}\|g\|_{\mathscr{Y}_{L^{n} / \varepsilon}(\beta)}
$$

with a constant $C$ which can be chosen independent of $L, \varepsilon$, and $n$. We obtain with (22) and (23) that

$$
N_{c}=s_{1}+s_{2}+\mathcal{O}\left(\frac{\varepsilon^{2}}{L^{3 n(1-2 p / 3)}}\left(\left\|v_{n}\right\|_{\mathscr{Y}_{L^{n} / \varepsilon}(2)}+\left\|w_{n}\right\|_{\mathscr{Y}_{L^{n} / \varepsilon}(2)}\right)^{2}\right)
$$


where

$$
\begin{aligned}
& s_{1}=-3 \varepsilon^{2} L^{-n} E_{c n} \tilde{u}_{0} v_{n} * v_{n}, \\
& s_{2}=-6 \varepsilon^{4} L^{-2 n} E_{c n}\left(\tilde{u}_{0} v_{n} *\left(\Lambda_{n}^{s}\right)^{-1} E_{s n}\left(3 \tilde{u}_{0} v_{n} * v_{n}\right)\right)-\varepsilon^{2} L^{-2 n} E_{c n} v_{n} * v_{n} * v_{n} .
\end{aligned}
$$

It remains to estimate $s_{1}$ and $s_{2}$. To obtain the required estimate for $s_{1}$ we need another $L^{(p-2) n}$ and for $s_{2}$ another $L^{(p-1) n}$. In Remark 8 we have already mentioned that in $x$-space one derivative gives $L^{-n}$. In Fourier space a multiplication with $i l$ gives also $L^{n}$. Due to (20) in $\mathscr{Y}_{L^{n} / \varepsilon}(2)$ the terms $s_{1}$ and $s_{2}$ are of the form

$$
\begin{aligned}
s_{2}= & {\left[\varepsilon^{2} L^{-2 n} \iint K_{2}\left(\varepsilon^{2} L^{-n} l, \varepsilon^{2} L^{-n}(l-m), \varepsilon^{2} L^{-n}(m-k), \varepsilon^{2} L^{-n} k\right)\right.} \\
& \left.\times a_{n}(l-m) a_{n}(m-k) a_{n}(k) d k d m\right] f_{1}(l), \\
s_{1}= & {\left[\varepsilon^{2} L^{-n} \int K_{1}\left(\varepsilon^{2} L^{-n} l, \varepsilon^{2} L^{-n}(l-m), \varepsilon^{2} L^{-n} m\right) a_{n}(l-m) a_{n}(m) d m\right] f_{1}(l), }
\end{aligned}
$$

where $v_{n}(l, x)=a_{n}(l) f_{1}\left(\varepsilon l / L^{n}, x\right)$. We have $K_{2}(0,0,0,0)=0$, since our coordinate transform (28) was chosen in such a way that in the spatial periodic case it is the quadratic approximation of the center manifold. Thus $\left|K_{2}(l, l-m, m-k, k)\right| \leqq$ $C(|l|+|l-m|+|m-k|+|k|)$ and so we gain another $L^{-n}$ using Lemma 14, i.e.

$$
\left\|s_{2}\right\|_{\mathscr{Y}_{L^{n} / \varepsilon}(1)} \leqq \frac{C \varepsilon^{2}}{L^{3 n}}\left(\left\|v_{n}\right\|_{\mathscr{Y}_{L^{n} / \varepsilon}(2)}+\left\|w_{n}\right\|_{\mathscr{Y}_{L^{n} / \varepsilon}(2)}\right)^{2}
$$

The projection $P_{1}(l)$ is orthogonal in $L^{2}(0,2 \pi)$ and given by $P_{1}(l) u=\left(\int \overline{f_{1}(l, x)}\right.$ $u(l, x) d x) f_{1}(l, \cdot)$. Thus

$$
K_{1}(l, l-m, m)=3 \int \overline{f_{1}(l, x)} f_{1}(l-m, x) f_{1}(m, x) \tilde{u}_{0}(x) d x .
$$

Using now Lemma 4 shows that

$$
\begin{aligned}
K_{1}(l, l-m, m)= & 3 \int\left[g_{0}(x) g_{0}(x) g_{0}(x) \tilde{u}_{0}(x)\right. \\
& -i \lg _{1}(x) g_{0}(x) g_{0}(x) \tilde{u}_{0}(x)+g_{0}(x) i(l-m) g_{1}(x) g_{0}(x) \tilde{u}_{0}(x) \\
& \left.+g_{0}(x) g_{0}(x) \operatorname{im} g_{1}(x) \tilde{u}_{0}(x)+\mathcal{O}\left(l^{2}+(l-m)^{2}+m^{2}\right)\right] d x .
\end{aligned}
$$

Note that $\tilde{u}_{0}(x)$ is an even function, and so $g_{0}(x)=\partial_{x} \tilde{u}_{0} /\left\|\partial_{x} \tilde{u}_{0}\right\|_{L^{2}(0,2 \pi)}$ is an odd function. By a translation of the problem, this can be assumed for all $u_{0}(\omega, \varphi, \varepsilon)$. Then the integral over the zero order terms goes finally over an odd function and vanishes. Since additionally the first order terms cancel we have

$$
\left|K_{1}(l, l-m, m)\right| \leqq C\left|l^{2}+(l-m)^{2}+m^{2}\right|
$$

Thus Lemma 14 shows us that

$$
\left\|s_{1}\right\|_{\mathscr{Y}_{L^{n} / \varepsilon}(2 p)} \leqq \frac{C \varepsilon^{2}}{L^{3 n(1-2 p / 3)}}\left(\left\|v_{n}\right\|_{\mathscr{Y}_{L^{n} / \varepsilon}(2)}+\left\|w_{n}\right\|_{\mathscr{Y}_{L^{n} / \varepsilon}(2)}\right)^{2}
$$


and eventually $\left\|N_{c}\right\|_{\mathscr{Y}_{L^{n} / \varepsilon}(2 p)} \leqq \varepsilon^{2} C R^{2} L^{-3 n(1-2 p / 3)}$. Hence

$$
\begin{aligned}
& \sup _{T \in\left[1 / L^{2}, 1\right]}\left\|\varepsilon^{-2} L^{2 n} \int_{1 / L^{2}}^{T} e^{L^{2 n} \varepsilon^{-2} \Lambda_{n}^{c}(T-\tau)} N_{c} d \tau\right\|_{\mathscr{Y}_{L^{n} / \varepsilon}(2)} \\
& \leqq C \varepsilon^{-2} L^{2 n} \varepsilon^{2} R^{2} L^{-3 n(1-2 p / 3)} \int_{1 / L^{2}}^{1}\left(T-1 / L^{2}\right)^{2 p-1} d T \\
& \leqq C L^{-n(2 p-1)} R^{2} .
\end{aligned}
$$

d) It remains to combine a), b) and c). Choosing $\delta$ and $R$ in the above way it is easy to see that the right-hand side of (30) defines a contraction in a ball of radius $2 C L^{5 / 2-\alpha}$ in $C\left(\left[1 / L^{2}\right], \mathscr{Y}_{L^{n} / \varepsilon}(2)\right)$ if $L$ is chosen sufficiently large. Therefore the solution which is the fixed point of this contraction stays in $\mathscr{Y}_{L^{n} / \varepsilon}(2)$ in a ball of the same radius.

Remark. 26. Since we have to use the smoothing properties (32) of the linear semigroup and since we have to integrate from $1 / L^{2}$ to 1 we have to choose $\beta=$ $2 p>0$.

Remark. 27. Let us remark about the general situation $u_{0}(\omega, \varphi, \varepsilon)$ that as shown in Lemma 4 the eigenfunctions $f_{1}$ and subsequently $K_{1}$ has expansions with the same properties. Consequently these estimates hold for all $u_{0}(\omega, \varphi, \varepsilon)$.

Proof of Lemma 25. a) and b) follow from the proof of the points a) and b) of Lemma 24. The point c) follows since $\mathscr{Y}_{L^{n} / \varepsilon}(2) \subset C_{b}^{0}\left(\left[-L^{n} /(2 \varepsilon), L^{n} /(2 \varepsilon)\right), H^{2}\right)$. Now, let $g \in \mathscr{Y}_{L^{n-1 / \varepsilon}}(2)$ with $\|g(l, \cdot)\|_{H^{2}} \leqq C|l|^{\beta}$, then

$$
\begin{aligned}
& \left\|e^{L^{2 n} \varepsilon^{-2} \Lambda_{n}^{c}\left(1-1 / L^{2}\right)} E_{c n}^{h} \mathscr{L}_{1 / L} g\right\|_{\mathscr{Y}_{L^{n} / \varepsilon}(2)} \\
& \quad \leqq\left\|K \mapsto e^{L^{2 n} \varepsilon^{-2} \mu_{1}\left(\varepsilon K / L^{n}\right)\left(1-1 / L^{2}\right)} \tilde{e}_{c n}^{h} g(k / L)\right\|_{\mathscr{Y}_{L^{n} / \varepsilon}(2)} \\
& \quad \leqq C L^{-\beta}\|g\|_{\mathscr{Y}_{L^{n-1_{\varepsilon}}(2)}} .
\end{aligned}
$$

Applying this to $\rho$ and using $\rho(K=0)=0$ shows the point $\mathrm{d})$. Applying Lemma 14 to $\left|e^{\mu_{1}\left(\varepsilon K / L^{n}\right) \varepsilon^{-2} L^{2 n}\left(1-1 / L^{2}\right)}-e^{-K^{2}\left(1-1 / L^{2}\right)}\right| \leqq C|K|$ shows the point e). The point f) follows since the spectrum of $E_{s}^{h} \Lambda$ is below $-C \varepsilon^{2}$.

Acknowledgements. The author is grateful for discussions with P. Collet, J.-P. Eckmann, Th. Gallay, and B. Sandstede. This work was stimulated by visits at the University of Geneva which were sponsored by the Fonds National Suisse. Moreover, it was partially supported by the Deutsche Forschungsgemeinschaft DFG under the grant Mi 459/2-1.

\section{References}

[BK92] Bricmont, J., Kupiainen, A.: Renormalization group and the Ginzburg-Landau equation. Commun. Math. Phys. 150, 193-208 (1992)

[BK94] Bricmont, J., Kupiainen, A.: Stability of moving fronts in the Ginzburg-Landau equation. Commun. Math. Phys. 159, 287-318 (1994)

[CE87] Collet, P., Eckmann, J.-P.: The stability of modulated fronts. Helv. Phys. Acta 60, 969991 (1987) 
[CE90a] Collet, P., Eckmann, J.-P.: Instabilities and Fronts in Extended Systems. Princeton, NJ: Princeton University Press, 1990

[CE90b] Collet, P., Eckmann, J.-P.: The time dependent amplitude equation for the SwiftHohenberg problem. Commun. Math. Phys. 132, 139-153 (1990)

[CE92] Collet, P., Eckmann, J.-P.: Solutions without phase-slip for the Ginsburg-Landau equation. Commun. Math. Phys. 145, 345-356 (1992)

[CEE92] Collet, P., Eckmann, J.-P., Epstein, H.: Diffusive repair for the Ginsburg-Landau equation. Helv. Phys. Acta 65, 56-92 (1992)

[Eck65] Eckhaus, W.: Studies in nonlinear stability theory. Springer tracts in Nat. Phil. Vol. 6, 1965

[Eck93] Eckhaus, W.: The Ginzburg-Landau equation is an attractor. J. Nonlinear Science 3, 329-348 (1993)

[EW94] Eckmann, J.-P., Wayne, C.E.: The non-linear stability of front solutions for parabolic partial differential equations. Commun. Math. Phys. 161, 323-334 (1994)

[Ga94] Gallay, T.: Local stability of critical fronts in nonlinear parabolic partial differential equations. Nonlinearity 7, 741-764 (1994)

[GJK93] Gardner, R., Jones, J.K.R.T., Kapitula, T.: Stability of travelling waves for nonconvex scalar viscous conservation laws. Comm. Pure. Appl. Math. 46, 505-526 (1993)

[Ha94] Haragus, M.: The orbital stability of fronts for high order parabolic partial differential equations. In: Structure and Dynamics of Nonlinear Waves in Fluids (eds.: A. Mielke, K. Kirchgässner) World Scientific Publishing 1995, 268-274.

[He81] Henry, D.: Geometric Theory of Semilinear Parabolic Equations. Lecture Notes in Mathematics 840, Berlin, Heidelberg, New York: Springer, 1981

[Ka94] Kapitula, T.: On the nonlinear stability of plane waves for the Ginzburg-Landau equation. Comm. Pure. Appl. Math. 47, 831-841 (1994)

[Ki92] Kirchgässner, K.: On the nonlinear dynamics of travelling fronts. J. Diff. Eqns. 96, 256-278 (1992)

[KSM92] Kirrmann, P., Schneider, G., Mielke, A.: The validity of modulation equations for extended systems with cubic nonlinearities. Proceedings of the Royal Society of Edinburgh 122A, 85-91 (1992)

[Mi94] Mielke, A.: A new approach to sideband-instabilities using the principle of reduced instability. In: Nonlinear Dynamics and Pattern Formation in the Natural Environment (eds.: A. Doelman, A. van Harten) Longman UK 1995, 206-222

[MS95] Mielke, A., Schneider, G.: Attractors for modulation equations on unbounded domains -Existence and comparison. Nonlinearity 8,743-768 (1995)

[NW69] Newell, A., Whitehead, J.: Finite bandwidth, finite amplitude convection. J. Fluid Mech. 38, 279-303 (1969)

[PW94] Pego, R.L., Weinstein, M.I.: Asymptotic stability of solitary waves. Commun. Math. Phys. 164, 305-349 (1994)

[RS72] Reed, M., Simon, B.: Methods of Modern Mathematical Physics 1-4. New York: Academic Press, 1972

[Sch94a] Schneider, G.: A new estimate for the Ginzburg-Landau approximation on the real axis. J. Nonlinear Science 4, 23-34 (1994)

[Sch94b] Schneider, G.: Error estimates for the Ginzburg-Landau approximation. J. Appl. Math. Physics (ZAMP) 45, 433-457 (1994)

[Sch94c] Schneider, G.: Global existence via Ginzburg-Landau formalism and pseudo-orbits of Ginzburg-Landau approximations. Commun. Math. Phys. 164, 157-179 (1994)

[Sch95] Schneider, G.: Analyticity of Ginzburg-Landau modes. J. Diff. Eqns. 121, 233-257 (1995)

[Te88] Temam, R.: Infinite-Dimensional Systems in Mechanics and Physics. Berlin, Heidelberg, New York: Springer, 1988

[vH91] van Harten, A.: On the validity of Ginzburg-Landau's equation. J. Nonlinear Science 1, 397-422 (1991)

[Wa94] Wayne, E.C.: Invariant manifolds for parabolic partial differential equations on unbounded domains. Preprint Pennsylvannia State University, 1994. To appear in Arch. Rat. Mech. 1996 